\title{
PRINCIPLES OF RADIO TRANSMISSION AND RECEP. TION WITH ANTENNA AND COIL AERIALS
}

\author{
By J. H. Dellinger
}

CONTENTS

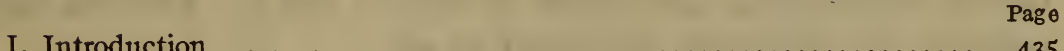

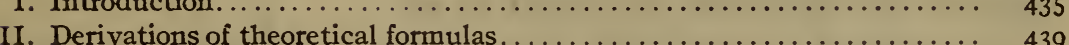

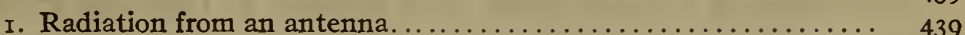

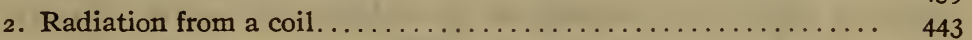

3. Received current in an antenna. . . . . . . . . . . . . . . . 446

4. Received current in a coil......................... 448

III. Discussion of theory of radiation and reception . . . . . . . . . . $45^{2}$

I. Distinction between induction and radiation . . . . . . . . . $45^{2}$

2. Is radiation limited to high frequencies? . . . . . . . . . . 453

3. Equivalence of electrostatic and magnetic fields in a wave..... 454

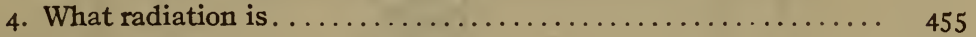

IV. Comparison formulas................................... 456

I. Derivation from theoretical formulas.................. $45^{6}$

2. Examples of comparison of coil and antenna.............. $45^{8}$

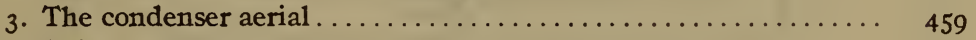

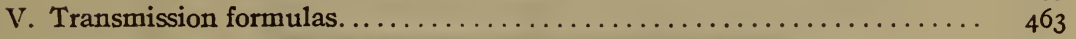

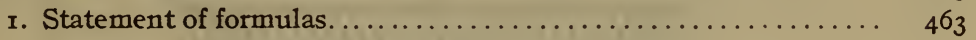

2. Discussion of transmission formulas................. 464

VI. Experimental verification of formulas.................... 467

I. Principles of measurement of received current and voltage, with applications to design.......................... 467

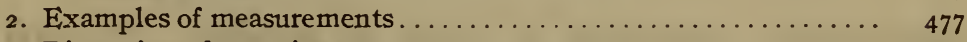

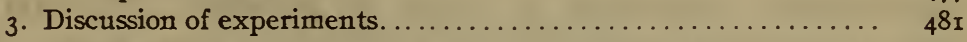

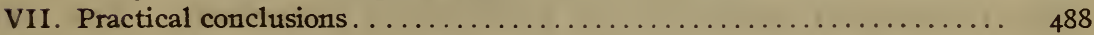

I. Relative effectiveness of antennas and coil aerials ......... 488

2. Principal formulas.......................... 489

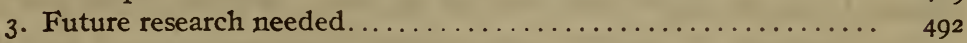

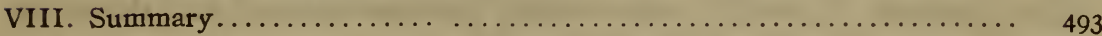

\section{INTRODUCTION}

In a radio transmitting or receiving set, either the condenser or the inductance coil is made of large dimensions. It is then called the aerial, and effects the transfer of power between the radio circuits and the ether. The coil aerial has the inherent advantage of serving as a direction finder and interference preventer, but is less effective quantitatively as a transmitting or 
receiving device than the condenser type of aerial, commonly called the antenna. Both kinds of aerial are very simple in construction, consisting merely of one or more wires. An antenna consists of a wire or set of wires connected in parallel and constituting one plate of a condenser, the other plate being the ground beneath. The coil aerial is one or more turns of wire constituting a simple coil or loop. When an antenna is used its circuit is completed, in general, by placing an inductance coil in series with it and the ground; and when a coil aerial is used its circuit is completed by connecting a condenser across its terminals. The typical connections are shown in Figs. I and 2. The coil aerial requires no connection to ground at all.

The antenna is used when it is desired to communicate over as great a range as possible or to reduce the power required in the

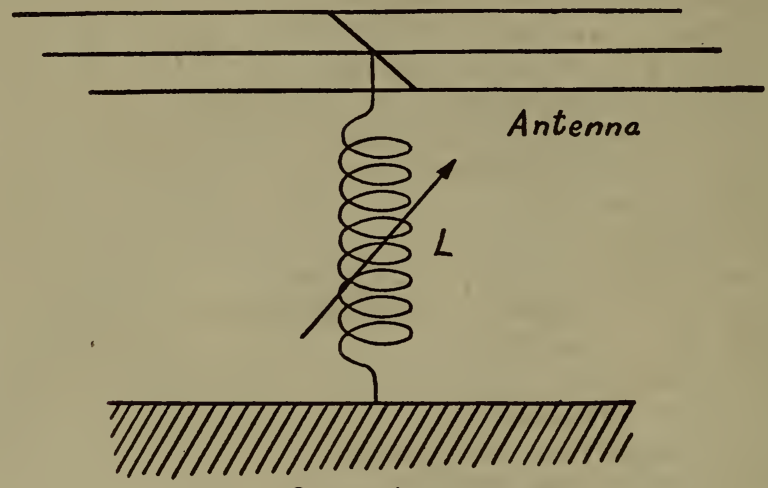

Ground

FIG. I.-Simple antenna circuit

transmitting apparatus as much as possible. The coil is used when directional properties are particularly important. The coil radiates and receives electric waves better in the direction of its plane than in the direction of its axis, whereas the performance of an antenna is much more nearly independent of the direction of the waves. By arranging a coil so that it can be rotated it makes an excellent direction finder. When thus used on a ship or an airplane a coil aerial is sometimes called a radio compass. It has also been called a radio goniometer. By turning it so that its axis is parallel to the direction of propagation of the wave from some particular station, that wave is not received while waves from other directions are received. The coil may thus serve as an interference preventer. It is possible to attain some slight reduction of the effects of strays, commonly called static, 
by using combinations of coil aerials. Submarine communication is more successful with coil aerials than with antennas, because the coil can be protected from the short-circuiting effect of the water while an antenna can not. The numerous advantages of the coil aerial make it highly important to know the relative sensitiveness or power of transmission of the device in comparison with the antenna. This publication provides the answer to this

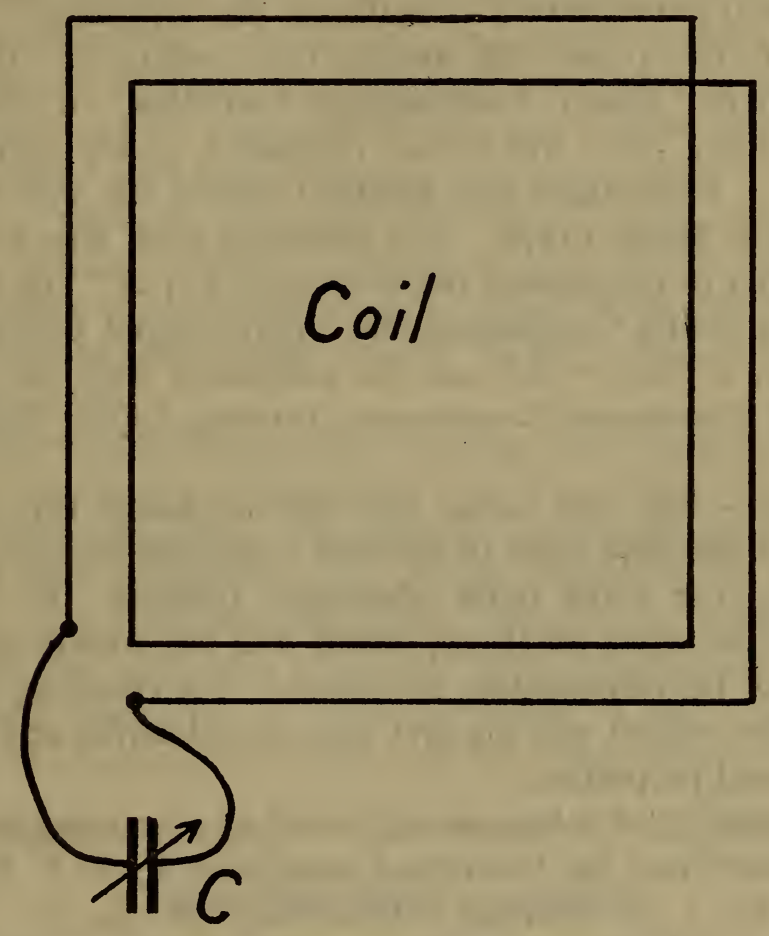

FIG. 2.- Simple coil aerial circuit

question and sets forth the theory of radiation and reception and the action of antenna and coil aerials. The relative effectiveness of any coil and antenna is given by formulas (32) to (36) in Section IV, I, below. The uses of the coil as a direction finder, interference preventer, reducer of strays, and submarine aerial, are not treated in this article.

The most important question considered is the practical one: How far can communication be maintained by the use of any specified antennas or coil aerials? Formulas are developed by which the current received in an antenna or coil is calculated in terms of the current in a transmitting antenna or coil, resistance of receiving aerial circuit, the distance, wave length, and 
dimensions of the aerials. The formulas have been found to be useful in the design of aerials and in the selection of an aerial for a particular kind of communication. They were worked out before there was any experimental information available to answer the question of the comparative quantitative value of the two kinds of aerials. Not much information on this has been obtained from experiment even yet (1919), but such experiments as have been made have substantiated the formulas. The work described in this paper was done in 1916 and 1917. The results were given in "Radio Transmission Formulas," a confidential paper of July, I917, which was circulated in the Signal Corps and Navy. Publication was withheld during the war at the request of the Signal Corps. The formulas have also been given by the writer of the present paper on page 234 of "The Principles Underlying Radio Communication," r918, Signal Corps Pamphlet No. 40, a book which can be purchased from the Superintendent of Documents, Government Printing Office, Washington, D. C.

Historical.-The coil aerial and the condenser type of aerial (antenna) both date back to the first experimenter with the electric waves that make radio telegraphy possible. H. Hertz, in r 888, used an open oscillator, which was the forerunner of the antenna, as his transmitting apparatus. For receiving he used a circle of wire, which was the first loop or coil aerial, and observed its directional properties.

The possibility of a loop or coil aerial as a transmitting device was discussed from the theoretical standpoint by G. F. Fitzgerald and later by J. A. Fleming (Electrician, vol. 59, pp. 936, 976, ror6; 1907). Fleming derived expressions for the radiated fields, using a curious theory in which the four sides of the coil were replaced by Hertzian doublets.

The use of a large loop or single-turn coil as an aerial in practical radio communication was described by G. Pickard (Proceedings of the Wireless Institute of America, vol. I, May, I, 1909). He discussed its properties both as a radiating and receiving aerial. He described its use as a direction finder, stating that he had determined directions with it to better than I degree.

In spite of this work and proposals by others, the antenna was used almost exclusively as the transmitting and receiving device until I9I3. The use of the coil aerial received a great impteus by the publication of an article by $F$. Braun (Jahrbuch der drahtlosen Telegraphie und Telephonie, vol. 8 , p. I; 19I4), on the Use 
of Closed Circuits in Place of Open in Radio Telegraphy. $\mathrm{He}$ discussed the advantages of a coil aerial as a receiver and transmitter, both from the theoretical and the experimental standpoint.

Since 1913 there has been a great deal of development work done on coil aerials and they were extensively used in the war. The development of the coil aerial as a practical direction finder and receiving device was begun at the Bureau of Standards in 1915. Using electron tubes as the detecting apparatus, transAtlantic signals were received on a coil inside a room. Experiments with the coil as a transmitting device were carried out at the Bureau in 1917. Among the very few published treatments of development and use of the coil aerial are those in Bucher's textbook "Practical Wireless Telegraphy," I917, p. 256; and "Radio Direction-Finding Apparatus," by A. S. Blatterman (Elec. World, vol. 73, p. 464; 1919). Most of the descriptions to date have been confidential reports of the military services of various countries.

The theoretical discussions by Fleming and Braun are cumbersome and needlessly complicated and the results are not well adapted to practical use. The present paper presents an original treatment that is relatively very simple, but none the less exact, and leads to conclusions that apply directly to practical work. This paper also points out a number of misconceptions that have existed, and endeavors to clear up some of the controversial points on the radiation of waves and the functioning of aerials.

\section{DERIVATIONS OF THEORETICAL FORMULAS}

\section{RADIATION FROM AN ANTENNA}

Formula (8) below, giving the radiated magnetic field at a distance from an antenna, is a well-known formula. It has been given by various writers, and is the only one presented in this paper that requires any deep consideration of fundamental electromagnetic theory. The result is in fact implicit in Maxwell 's classical treatise, "Electricity and Magnetism." The derivation given here is much more direct and brief than the others the author has seen, and is given only for that reason. The derivations of formula (ro) and following ones are still simpler, and will be of more interest to most readers.

The units used in this paper are international electric units, the ordinary electric units based on the ohm, ampere, centimeter, and second. (See paper by the author on "International System 
of Electric and Magnetic Units," Scientific Paper of the Bureau of Standards No. 292.) The unit of magnetic field intensity is the gilbert per $\mathrm{cm}$, often called the cgs unit. The only exception to the use of units of the international system is in certain of the practical formulas where lengths are expressed in meters or miles where so stated.

In the following discussion is calculated the magnetic field intensity produced by a flat-top antenna, having electric current of uniform value throughout the length of the vertical portion. Most antennas in practice approximate closely this condition. The symbols used are:

$i=$ instantaneous current.

$I_{\mathrm{o}}=$ maximum value of current.

$I=$ effective value of current.

$H_{t}=$ instantaneous value of magnetic field intensity.

$H_{\mathrm{o}}=$ maximum value of magnetic field intensity.

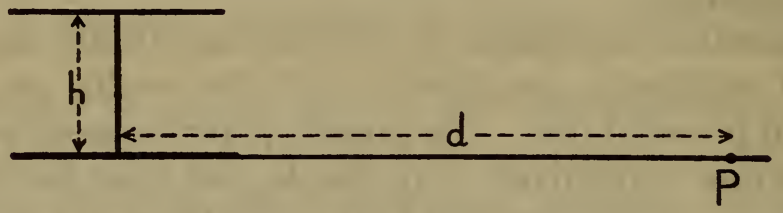

FIG. 3.-Calculation of magnetic field at a distance from an antenna

$H=$ effective value of magnetic field intensity.

$h=$ height of aerial.

$d=$ distance from sending aerial.

$\omega=2 \pi$ times frequency of the current.

$t=$ time.

$\lambda=$ wave length.

$c=$ velocity of electric waves $=3 \times 10^{10} \mathrm{~cm}$ per second.

Subscripts $(:) \mathrm{s}=$ sending, $\mathrm{r}=$ receiving, $\mathrm{a}=$ antenna, $\mathrm{c}=\mathrm{coil}$.

In Fig. 3 the upper heavy line represents the flat top of the antenna and the lower heavy line the grounding area. Suppose a current is flowing having the instantaneous value $i$ in the vertical portion. The magnetic field intensity at any point due to a varying current is different from that due to a steady current. Consequently the field can not be calculated in the same way that the magnetic field intensity of a straight wire is ordinarily calculated. When the current is varying, the magnetic field intensity is calculated by the aid of a quantity called the vector potential in such a way that the variation with time is taken into account. The instantaneous value of the vector potential of current in the 
vertical conductor at a distance $d$ in a plane perpendicular to the conductor, is

$$
A=\frac{[i] h}{d}
$$

where [i] indicates that for any time $t$ the value of $i$ is taken for the instant $\left(t-\frac{d}{c}\right)$.

Suppose the current in the antenna is a sine-wave alternating current,

$$
\begin{gathered}
i=I_{\mathrm{o}} \sin \omega t \\
\therefore[i]=I_{\mathrm{o}} \sin \omega\left(t-\frac{d}{c}\right) \\
A=\frac{h[i]}{d}=\frac{h I_{\mathrm{o}}}{d} \sin \omega\left(t-\frac{d}{c}\right)
\end{gathered}
$$

The magnetic field intensity is calculated from the vector potential by the general relation $H_{\mathrm{t}}=0$.I curl $A$, which for this simple case of a straight conductor becomes

$$
H_{\mathrm{t}}=\frac{\mathrm{I}}{\mathrm{IO}} \frac{\partial A}{\partial d},
$$

the direction of $H_{\mathrm{t}}$ being perpendicular to the plane of $h$ and $d$. From equation (3),

$$
H_{\mathrm{t}}=-\frac{h \omega I_{\mathrm{o}}}{\mathrm{IOcd}} \cos \omega\left(t-\frac{d}{c}\right)-\frac{h I_{\mathrm{o}}}{\mathrm{IOd}^{2}} \sin \omega\left(t-\frac{d}{c}\right)
$$

This equation gives the magnetic field intensity at any point $P$ at a distance $d$ from the antenna. The second term represents the ordinary induction field associated with the current, while the first term is the radiation field. At a considerable distance the second term is negligible, because the second power of $d$ occurs in the denominator. The first term then represents the magnetic field radiated from an antenna at the distance $d$ from the antenna. The distance $d$ is measured along the earth's surface, because the waves follow the curvature of the earth's surface instead of proceeding straight out into space. For a considerable distance from the antenna the maximum value of the magnetic field intensity during a cycle is therefore

$$
H_{\mathrm{o}}=\frac{h \omega I_{\mathrm{o}}}{\mathrm{IOcd}}
$$


Expressing in terms of effective values,

$$
H=\frac{h \omega I}{\mathrm{IOcd}}
$$

Henceforth $H$ means the radiated field, unless it is specifically stated to be the total field. The last equation may be expressed in terms of wave length instead of $\omega$ by the relation

$$
\begin{aligned}
\frac{\omega}{c} & =\frac{2 \pi}{\lambda} \\
\therefore H & =\frac{2 \pi}{\mathrm{IO}} \frac{h I}{\lambda d}
\end{aligned}
$$

Using the subscript $s$ to indicate that it is the sending rather than the receiving antenna which is considered,

$$
H=\frac{2 \pi}{10} \frac{h_{\mathrm{s}} I_{\mathrm{s}}}{\lambda d}
$$

This derivation follows the conceptions presented in the early pages of Lorentz, "The Theory of Electrons." It is equivalent to Hertz's intricate proof, but is more direct. The way in which the result is expressed here accords more closely with the physical ideas and with actual practice, being expressed in terms of current rather than electric charge, since it is current that is actually measured in an antenna, and the current, furthermore, is generally uniform in the vertical portion of the antenna.

Formula (8) gives the radiated magnetic field from a sending antenna at a distance $d$ along the earth's surface. The units are the gilbert per $\mathrm{cm}$ for $H$, the ampere for $I$, and the centimeter for all lengths, as previously stated.

Undamped alternating current in the antenna was assumed. The same result, however, is obtained if the current is damped.

At very great distances from the sending aerial, the magnetic field is less than that calculated by formula (8), because of absorption of the power of the wave in the ground as it travels along. This may be taken into account by multiplying the right-hand member of (8) by a correction factor $F_{1}$. The value of this factor for daytime transmission over the ocean, derived from the experiments of L. W. Austin, Scientific Paper of the Bureau of Standards No. I59; I9II, is

$$
F_{1}=\epsilon^{-0.000047 \frac{d}{\sqrt{\lambda}}}
$$

for $d$ and $\lambda$ both in meters. This correction ordinarly needs to be applied only when the distance is greater than roo kilometers. 


\section{RADIATION FROM A COII}

It was formerly the belief that a coil could not radiate, because the current up one side of the coil (Fig. 4) produces a field equal and opposite to that down the other side of the coil. This is erroneous because the two equal fields are not exactly opposite. The phase between the two departs from $180^{\circ}$ because of the finite time required for the field to be propagated from one side of the coil to the other. It is only along the axis of the coil that the calculated radiation is zero. The actual resultant field radiated from the coil may be deduced in either of two very simple ways, both of which are interesting from the physical

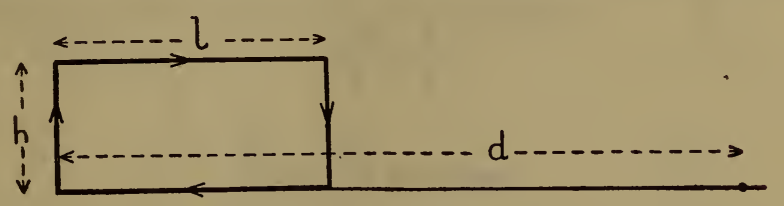

Fig. 4.-Calculation of magnetic field radiated from a coil

standpoint. The first deals with the instantaneous values of the magnetic field, and the second with the effective values.

The following additional symbols are used:

$l=$ horizontal length of coil aerial.

$N=$ number of turns of wire of coil aerial.

$\theta=$ phase angle between values of field intensity a distance $l$ apart in the wave.

First Deduction.-Consider a rectangular coil of height $h$ and horizontal length $l$. The magnetic field at a point $P$ in the $d$ direction is the resultant of the fields arising from current in the two vertical sides of the coil. The two horizontal sides contribute nothing, because a point at great distance $d$ is practically equidistant from these two sides. The magnetic field due to any one of the vertical wires of the coil is calculated from equation (5) above. Neglecting the second term, because $d$ is large, the instantaneous values of the magnetic field (Fig. 4) at the distances $d$ and $(d-l)$, respectively, from the two vertical sides are

$$
\begin{gathered}
H_{\mathrm{d}}=-\frac{h N \omega I_{\mathrm{o}}}{\text { IO } c d} \cos \omega\left(t-\frac{d}{c}\right) \\
H_{\mathrm{d}-1}=+\frac{h N \omega I_{\mathrm{o}}}{\mathrm{IO} c(d-l)} \cos \omega\left(t-\frac{d-l}{c}\right)
\end{gathered}
$$


The resultant field $H_{\mathrm{t}}$ is the algebraic sum of these two, which becomes, since $(d-l)$ is very nearly $d$ when $d$ is !arge,

$$
H_{\mathrm{t}}=-\frac{h N \omega I_{\mathrm{o}}}{\mathrm{ro} c d} 2 \sin \omega\left(t-\frac{d-\frac{l}{2}}{c}\right) \sin \frac{\omega l}{2 c}
$$

The effective value of the resultant field is

$$
H=\frac{2}{10} \frac{h N \omega I}{c d} \sin \frac{\omega l}{2 c}
$$

Using the relations

$$
\begin{gathered}
\frac{\omega}{c}=\frac{2 \pi}{\lambda} \\
\text { and } \sin \frac{\omega l}{2 c}=\frac{{ }^{\prime} \omega l}{2 c}
\end{gathered}
$$

the latter holding when the angle is small; that is, $l$ small compared with the wave length,

$$
H=\frac{4 \pi^{2}}{I_{0}} \frac{h_{\mathrm{s}} l_{\mathrm{s}} N_{\mathrm{s}} I_{\mathrm{s}}}{\lambda^{2} d}
$$

This is the radiated magnetic field from a sending coil aerial at a distance $d$ along the earth's surface, the direction of $d$ being the plane of the coil. The units are international units as stated. under equation (8). The deduction assumes that the ground below the coil is not so good a conductor as to form an image of the coil. Thus the formula applies to a radiating coil in an airplane as well as to one at a ground or ship station.

The formula applies for either damped or undamped current $I_{\mathrm{s}}$ in the sending antenna. For very great distances the righthand side of the formula must be multiplied by the distance correction factor $F_{1}$ given in (9), the same as for a radiating antenna.

Second Deduction.-The radiated magnetic field due to one of the sides of the coil is $N_{\mathrm{s}} H_{1}$, and from formula (8),

$$
H_{1}=\frac{2 \pi}{10} \frac{h_{\mathrm{B}} I_{\mathrm{s}}}{\lambda d}
$$


If the two vertical sides of the coil coincide, their magnetic fields would be equal and opposite, as shown by the lines $O A$ and $O B$, Fig. 5. But since the two vertical sides are separated by the distance $l$, at any instant the field a distance $d$ (Fig. 4) from the left side of the coil has traveled a distance $l$ farther than the field from the right side. If then $N_{8} H_{1}$ is the field due to the right side, the field at the same point due to the left side is shifted in phase from the position $O B$ to $O C$ in Fig. 5, where the angle $\theta$ between them is the phase angle between the values of the field a distance $l$ apart in the waves.

The distance $l$ is the same fraction of the wave length that the angle $\theta$ is of a complete cycle, $2 \pi$. That is,

$$
\frac{\theta}{2 \pi}=\frac{l_{\mathrm{B}}}{\lambda}
$$

or

$$
\theta=2 \pi \frac{l}{\lambda}
$$

The resultant of $O A$ and $O . C$ is their vector sum,

$$
H=\dot{N}_{\mathrm{s}} H_{1} \sqrt{2(\mathrm{I}-\cos \theta)}
$$

When $\theta$ is small, i. e., $l$ small compared with the wave length,

$$
\begin{gathered}
\frac{H}{N_{8} H_{1}}=\sin \theta=\theta \\
\therefore H=N_{8} H_{1} \theta
\end{gathered}
$$

Thus the radiated magnetic field from a coil is equal to the field from one side of the coil

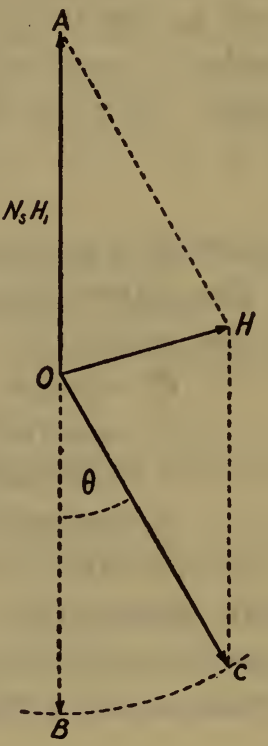

Fig. 5.-Phase relations of magnetic fields radiated from a coil multiplied by the phase angle $\theta$ corresponding to the distance $l$ between the sides of the coil.

From (I 4 ) and (II),

$$
H=\frac{2 \pi}{\mathrm{IO}} \frac{h_{\mathrm{s}} N_{\mathrm{s}} I_{\mathrm{s}}}{\lambda d} \theta
$$

This equation, together with (I 2), gives identically formula (Io) obtained by the first deduction.

It was assumed in these deductions that the current was uniform throughout the coil. If the distributed capacity of the coil is appreciable the current in the coil will be different at different points. Thus the current in the middle may be greater than at the ends. This also may give rise to radiation from the coil, but is an entirely separate phenomenon from the phase angle between 
the two sides of the coil which has been discussed. This question of distributed capacity requires consideration particularly when coils are used having dimensions comparable with the wave length. The phenomenon is discussed further under "Antenna effect" in section VI, 3 , below.

\section{RECEIVED CURRENT IN AN ANTENNA}

The current flowing in the receiving aerial circuit when the field intensity of the wave traversing the aerial is known can be calculated in several ways. An electromagnetic wave in space has both an electric and a magnetic field intensity which are at right angles to each other and to the direction of propagation of the wave. The two field intensities are related to each other by

$$
\mathbb{E}=300 . H
$$

where $\mathbb{E}^{\mathbb{E}}$ is in volts per $\mathrm{cm}$ and $H$ in gilberts per $\mathrm{cm}$.

The following additional symbols are used in this and the following section:

$\mathfrak{E}=$ electric field intensity.

$E=$ electromotive force in receiving aerial:

$R=$ resistance of receiving aerial circuit.

$\phi=$ magnetic flux.

First Deduction.-The electromotive force, and thence the current, produced in an antenna may be calculated from the principle that relative motion of a magnetic field and a conductor create an electromotive force in the conductor whose value is

$$
E=\mathrm{IO}^{-8} h_{\mathrm{r}} H c
$$

when the directions of the field, the motion, and the conductor are mutually perpendicular, $h_{\mathrm{r}}$ being the length of the conductor and $c$ the velocity of relative motion. This then gives the emf in an antenna of height $h_{\mathrm{r}}$, produced by an electromagnetic wave having magnetic field intensity $H$ and traveling with the velocity $c$.

In ordinary practice, the reactance in series with the antenna is varied to produce resonance to the frequency of the incoming wave, so that

$$
I_{\mathrm{r}}=\frac{E}{R}
$$

Inserting for $c$ its value, $3 \times 10^{10}$ in equation ( 17 ),

$$
I_{\mathrm{r}}=300 \cdot \frac{h_{\mathrm{r}} H}{R}
$$


This is the current in amperes received in a flat-top antenna, using the centimeter as the unit of length, with resistance of circuit in ohms, and the magnetic field intensity in gilberts per $\mathrm{cm}$.

The received current is less than that given by the formula if the wave is damped, since an undamped alternating field was assumed in the discussion. For a damped field the emf acting on the aerial is similarly damped and equation (I8) does not hold. Correct results are obtained by multiplying the right-hand side of formula (19) by the correction factor $F_{2}$ obtained as follows:

If the magnetic field intensity, and hence the emf, has the decrement $\delta^{\prime}$, the effective current is not $I_{\mathrm{r}}$, defined by (I8), but another value which we shall call $I_{\rho}$. The value of $I_{\rho}$ may be found by the aid of the generalized definition of decrement given in the author's paper, "The Measurement of Radio-Frequency Resistance, Phase Difference, and Decrement" (Proc. I. R. E., vol. 7, p. 27; Feb., 1919).

For decrements smaller than about 0.2 , the logarithmic decrement is one-half the ratio of the average energy dissipated per cycle to the average energy associated with the current at the maximum of the cycle.

Taking the average power as $\frac{E^{2}}{R}$, the average energy dissipated per cycle $=\frac{E^{2}}{f R}$. The average energy associated with the current at the maximum of each cycle $=L I_{\rho}{ }^{2}$. The energy-ratio definition of decrement just given applies to the sum of the decrements acting, viz, the decrement $\delta^{\prime}$ of the emf and the decrement $\delta$ of the aerial circuit. The value of $\delta$ is $\frac{R}{2 f L}$. Applying the decrement definition:

$$
\begin{aligned}
\delta^{\prime}+\delta & =\frac{\frac{E^{2}}{f R}}{2 L I_{\rho}^{2} .} \\
& =\frac{E^{2} R}{2 f L R^{2} I_{\rho}^{2}} \\
& =\frac{E^{2} \delta}{R^{2} I_{\rho}^{2}} \\
I_{\rho}^{2} & =\frac{E^{2}}{R^{2}} \frac{\delta}{\delta^{\prime}+\delta}
\end{aligned}
$$


From the relation, $\frac{E^{2}}{R^{2}}=I_{\mathrm{r}}^{2}$,

$$
I_{p}=I_{\mathrm{r}} \sqrt{\frac{\delta}{\delta^{\prime}+\delta}}=I_{\mathrm{r}} \sqrt{\frac{\mathrm{I}}{\mathrm{I}+\frac{600 \cdot L \delta^{\prime}}{R \lambda}}}
$$

where $L$ is in microhenries and $\lambda$ is in meters.

This reduces to $I_{p}=I_{\mathrm{r}}$ when $\delta^{\prime}$ is small compared with $\delta$. Thus in the particular case of an undamped wave, where $\delta^{\prime}=0$, no correction is needed.

Correct results are obtained from equation (I9) for any damped wave by multiplying its right-hand member by the correction factor $F_{2}$, given by

$$
F_{2}=\sqrt{\frac{\mathrm{I}}{\mathrm{I}+\frac{600 \cdot L \delta^{\prime}}{R \lambda !}}}
$$

where $L$ is the inductance of the receiving aerial circuit in microhenries and $\lambda$ is wave length in meters, and $\delta^{\prime}$ is the logarithmic decrement of the damped wave that is being received.

Second Deduction.-The same formula may be derived from entirely independent consideration of the electric field associated with the wave. The emf between two points in space is the product of the distance between them by the electric field intensity along the line joining them. Thus the emf produced in a flat-top antenna is $\mathbb{E}^{\mathbb{E}}$ times the height, the direction of $\mathbb{E}^{\mathbb{E}}$ being assumed to be vertical.

$$
E=h_{\mathrm{r}} \mathbb{E}^{Z}
$$

Inserting the value of $\mathbb{E}$ from (I6) and dividing by the resistance;

$$
I_{\mathrm{r}}=300 . \frac{h_{\mathrm{r}} H}{R}
$$

This is identically the same formula obtained above from consideration of the magnetic field.

\section{RECEIVED CURRENT IN A COIL}

The current in a receiving coil aerial can be calculated in a number of different ways, all very simple and all giving the same result. The first conception which will be presented is simply that an emf is produced in the circuit by the time variation of magnetic flux through it.

The other modes of calculation involve the phase angle between the two vertical sides of the coil. The emf's acting in the two 
vertical sides are exactly equal and oppose each other in producing a current around the circuit when the plane of the coil is perpendicular to the direction of propagation of the wave. When the coil is turned in any other direction, however, the emf's in the two sides are not exactly opposite in phase, because of the difference in time required for the field to be propagated to one side of the coil and to the other. The emf can be calculated either from the elecrtic or the magnetic field, as in the discussion of received current in an antenna: The resultant emf can be found either from the algebraic sum of the instantaneous emf's in the two vertical sides or the vector sum of the effective emf's. These two methods are used in the second and third deductions, respectively, below.

The phase angle between the two sides of the coil is a very different thing from the phenomenon caused by the distributed capacity of the coil. It is assumed in the deductions given here that the current is uniform in all parts of the coil, which is not true when the distributed capacity is appreciable. Such capacity is large in coils of dimensions comparable with the wave length, and in such cases consideration must be given to the separate and additional phenomenon of distributed capacity.

First Deduction.-Assuming that the dimensions of the coil are small compared with the wave length, the magnetic field intensity is practically uniform throughout the coil. When the plane of the coil is parallel to the direction of propagation of the wave, the emf induced in the coil is

Now,

$$
E=\mathrm{IO}^{-8} \omega \phi
$$

$$
\phi=\mu h_{\mathrm{r}} l_{\mathbf{r}} N_{\mathrm{r}} H
$$

Since the permeability $\mu=\mathrm{I}$, and $I_{\mathrm{r}}=\frac{E}{R}$ because in ordinary practice the condenser in series with the coil aerial is adjusted to produce resonance with the frequency of the incoming wave,

$$
\begin{aligned}
I_{\mathrm{r}} & =10^{-8} \frac{\omega \phi}{R}=10^{-8} \frac{\omega h_{\mathrm{r}} l_{\mathrm{r}} N_{\mathrm{r}} H}{R} \\
& =2 \pi c 10^{-8} \frac{h_{\mathrm{r}} l_{\mathrm{r}} N_{\mathrm{r}} H}{R \lambda} \\
I_{\mathrm{r}} & =600 . \pi \frac{h_{\mathrm{r}} l_{\mathrm{r}} N_{\mathrm{r}} H}{R \lambda}
\end{aligned}
$$


This is the current received in a rectangular coil aerial of $N$ turns, with its plane parallel to the direction of propagation of the wave. The units are international units, as stated under formula (19). No image is assumed in the ground, so the formula applies not only to a receiving coil at a ground or ship station, but also to an airplane direction finder, provided the airplane is not flying at so great a height that the field of the wave is appreciably different from its value at the ground.

There are two correction factors that may need to be applied to this formula, both of which make the result smaller. If the wave is damped, the right-hand side of the formula should be multiplied by the decrement correction factor $F_{2}$, given by (20), the same as for a receiving antenna.

When the plane of coil is in some direction other than parallel to the direction of propagation of the wave, the right-hand side of formula (23) must be multiplied by the direction correction factor $F_{3}$ given by

$$
F_{3}=\cos a
$$

where $a$ is the angle between the direction of propagation of the wave and the plane of the coil.

Second Deduction.-The emf produced in any one of the vertical wires of the coil is given by either equation ( $\mathrm{I} 7$ ) or (2I) above, deduced from considerations of the action of the magnetic and the electric field intensity, respectively. Each of these equations reduces to

$$
E_{1}=300 . h_{\mathrm{r}} H
$$

The instantaneous emf in either of the two vertical sides of the coil is therefore

$$
e^{\prime}=300 \cdot h_{\mathrm{r}} N_{\mathrm{r}} H_{\mathrm{o}} \cos \omega t
$$

The instantaneous emf in the other side of the coil is produced by the magnetic field existing in the wave a distance $l$ away, when the plane of the coil is parallel to the direction of propagation of the wave. This emf $e^{\prime \prime}$ has the same direction in space, but the opposite direction as far as producing current around the circuit is concerned.

$$
e^{\prime \prime}=-300 . h_{\mathrm{r}} N_{\mathrm{r}} H_{\mathrm{o}} \cos \omega\left(t-\frac{l}{c}\right)
$$

The resultant emf in the circuit is the algebraic sum of these two,

$$
e=300 \cdot h_{\mathrm{r}} N_{\mathrm{r}} H_{\mathrm{o}} 2 \sin \omega\left(t-\frac{l}{2 c}\right) \sin \frac{\omega l}{2 c}
$$


The effective value of the resultant emf is

$$
E=600 \cdot h_{\mathrm{r}} N_{\mathrm{r}} H \sin \frac{\omega l}{2 c}
$$

Since when the angle is small, that is, when $l$ is small compared with the wave length,

$$
\begin{aligned}
& \sin \frac{\omega l}{2 c}=\frac{\omega l}{2 c}=\pi \frac{l}{\lambda} \\
& E=600 \cdot \pi \frac{h_{\mathrm{r}} l_{\mathrm{r}} N_{\mathrm{r}} H}{\lambda}
\end{aligned}
$$

Dividing by $R$, this gives the identical value of $I_{\mathrm{r}}$ obtained in (23) by the first mode of deduction.

Third Deduction.- The emf produced in one of the sides of the coil is $N_{\mathrm{r}} E_{1}$, where from either equation ( $\mathrm{I}_{7}$ ) or (2I) above; that is, from consideration of either the magnetic or the electric field intensity, respectively,

$$
E_{1}=300 . h_{\mathrm{r}} H
$$

If the two vertical sides of the coil coincided, the emf's produced in them would be equal and exactly neutralize each other, as shown by the lines $O A$ and $O B$, Fig. 6. But since the two vertical sides are separated by the distance $l$, at any instant the field acting on one side of the coil has traveled a distance $l$ farther than that acting on the other side. If, then, $N_{\mathrm{r}} E_{1}$ is the emf in one side of the coil, the emf in the other side is shifted in phase from the position $O B$ to the position $O C$ in Fig. 6 , where the angle $\phi$ between them is the phase angle between the values of the field a distance $l$ apart in the wave.

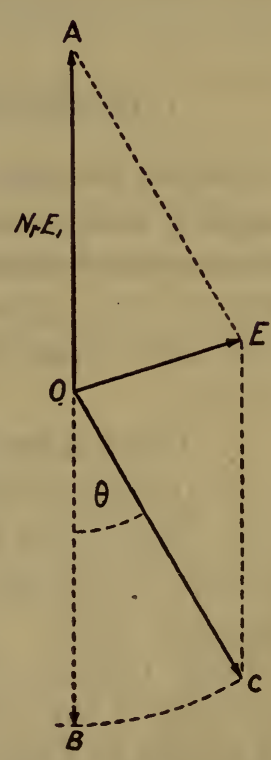

Fig. 6.-Phase relations of electromotive forces in receiving coil

The distance $l$ is the same fraction of the wave length that the angle $\theta$ is of a complete cycle, $2 \pi$; that is,

$$
\frac{\theta}{2 \pi}=\frac{l_{\mathrm{r}}}{\lambda}
$$

The resultant of $O A$ and $O C$ is their vector sum

$$
E=N_{\mathrm{r}} E_{2} \sqrt{2(\mathrm{I}-\cos \theta)} \text {. }
$$


When $\theta$ is small; i. e., $l$ small compared with $\lambda$

From (25),

$$
\begin{aligned}
\frac{E}{N_{\mathrm{r}} E_{1}} & =\sin \theta=\theta \\
\therefore E & =N_{\mathrm{r}} E_{1} \theta
\end{aligned}
$$

$$
E=300 \cdot h_{\mathrm{r}} N_{\mathrm{r}} H \theta
$$

Thus the emf acting in the coil is equal to the emf in one side of the coil multiplied by the phase angle $\theta$ corresponding to the distance $l$ between the sides of the coil.

Equations (27) and (30) combined give (26), and by dividing by $R$ formula (23) is obtained.

\section{DISCUSSION OF THEORY OF RADIATION AND RECEPTION}

\section{DISTINCTION BETWEEN INDUCTION AND RADIATION}

Certain fallacies which have appeared in textbooks and discussions arise from insufficient understanding of the difference between an induction field and a radiation field. Such fallacies are:

(a) An "open" circuit can radiate, while a "closed" circuit can not.

(b) There is no radiation from a circuit at low frequencies.

(c) Induction and radiation are the same phenomenon.

(d) The action of an antenna differs from that of a coil aerial in that the former is due to electrostatic fields and the latter to magnetic fields.

These fallacies will now be discussed. Fallacy $c$ has led to the supposition that the radiation and reception of electric waves can be taught in terms of transformer action. It should not be difficult to separate the two ideas, for there is a definite and clear distinction between the field due to induction and that due to radiation. The total magnetic field at a distance $d$ from a radiating antenna is, from equation (5)

$$
H=\frac{2 \pi}{10} \frac{h_{\mathrm{s}} I_{\mathrm{s}}}{\lambda d}+\frac{j}{10} \frac{h_{\mathrm{s}} I_{\mathrm{s}}}{d^{2}}
$$

where $j$ indicates that the two terms differ in phase by $90^{\circ}$. The first term represents the radiation field and the second term the induction field. The fact that one contains $\lambda d$ in the denominator 
while the other contains $d^{2}$ makes them radically different in nature. This gives the mathematical distinction between induction and radiation. The physical difference is discussed in section 3 below. It should be noted that the word "induction" here means not the production of an emf, but the magnetic induction, or flux per $\mathrm{cm}^{2}$, associated with a circuit.

The radiation field becomes relatively more important than the induction field as the distance $d$ is increased or as the wave length is diminished (i. e., the frequency increased). The question whether radiation or induction predominates in any given case can be settled by calculation from the formula. Thus, the two fields are equal at a distance

$$
d=\frac{\lambda}{2 \pi} .
$$

For points closer to the antenna than this the induction field predominates. For points farther away the radiation field predominates and the induction field falls off rapidly with distance and becomes negligible.

Certain early experiments in wireless signalling used true induction, e. g., the induction telegraphy of Preece and of Dolbear. When higher frequencies were used by later experimenters, signals of appreciable strength were received at distances of several wave lengths. These were genuine radiation signals, now commonly called radio.

\section{IS RADIATION LIMITED TO HIGH FREQUENCIES?}

The answer to this should be obvious from formulas (8) and (10). The radiated field does not become zero, no matter how great $\lambda$ is. For alternating current of any frequency, no matter how low, radiation takes place from the circuit. To be sure, the radiation is greater the higher the frequency, so that high-frequency circuits are better radiators than low-frequency ones, and this is all the basis there is for the mistaken idea that only highfrequency circuits radiate.

This applies to radiation from a coil as well as from an antenna. It has sometimes been stated that a coil will not radiate, the statement being put in the form that only "open". circuits radiate. The statement is doubly faulty since electricity can flow only in closed circuits. The meaning intended by "open" circuit is a circuit containing a condenser of open form; that is, with two plates well separated. There are two misconceptions at the base of the belief that a "closed" circuit or one not containing a con- 
denser would not radiate. In the first place, some have doubtless thought that waves would be started in the ether only by an electrostatic disturbance and thus could not be produced by a metallically closed circuit. Or, supposing it was understood that a magnetic disturbance in the ether would send out a wave just as readily as an electrostatic disturbance, it may have been thought that the radiation from one side of the circuit would be neutralized from that from the opposite side. As has already been shown in this paper the two disturbances do not exactly neutralize each other, on account of the finite time of propagation from one side of the circuit to the other, and the resultant is what gives rise to the radiation from a metallically closed circuit.

\section{EQUIVALENCE OF ELECTROSTATIC AND MAGNETIC FIELDS IN A WAVE}

The physical distinctions between radiation and induction are (a) the latter can be fixed in space and the former moves through space with the velocity of light, and $(b)$ in the case of radiation the magnetic field is always accompanied by an electrostatic field of value

$$
\mathfrak{E}=300 . \quad H
$$

and vice versa, whereas in the case of induction there is no fixed relation. It is of course true that whenever magnetic induction varies an electrostatic field is produced, and similarly whenever electrostatic induction varies a magnetic field is produced. But it is only in a radiated wave that these variations take place in such a way that one can be calculated from the other by the fixed relation ( 16 ). When there is a fixed electrostatic field associated with a circuit which does not vary, the magnetic field associated with this electrostatic field is zero, and vice versa.

In a radiated wave, then, the electrostatic and magnetic field are no longer independent phenomena but are strictly equivalent. Indeed, they are but two aspects of the same thing. Perhaps this will be clearer from the analogy of a sound wave. In a mechanical apparatus, elastic action and inertia act independently in various parts of the apparatus. In a sound wave, however, the effects of elastic action and inertia are mutual parts of a single phenomenon, the sound wave.

It is possible to separate the electrostatic and magnetic induction fields associated with a circuit by an arrangement of magnetic shields, but the electrostatic and magnetic fields in a radiated wave can not be separated. (This should not be confused with the sepa- 
ration of antenna effect and coil effect discussed in Sec. VI, 3, below, under "Antenna Effect.")

In considering any effect of the electromagnetic wave, it is equally permissible to consider the electrostatic or the magnetic field associated with the wave. They are equivalent and lead to the same result. This has been amply demonstrated above in this paper. The current received in an antenna, calculated from the electrostatic field, was exactly the same as calculated from the magnetic field. The same agreement was found for the coil aerial. This disposes of the question whether the current produced in an antenna or a coil aerial is caused by the electrostatic or the magnetic field present in the wave or both.

Complete discussions of electromagnetic waves are given in such treatises as Maxwell, "Electricity and Magnetism," I873; Jeans, "Electricity and Magnetism," I907; Lorentz, "The Theory of Electrons," I 909.

\section{WHAT RADIATION IS}

It has been shown that radiation differs from induction by a definitely calculable amount, that either kind of circuit radiates at any frequency, that there are both an electrostatic and a magnetic field present in every wave, having a constant ratio, and that any effect of the wave may be considered as due either to the electrostatic or the magnetic field of the wave.

Radiation is the moving disturbance of the ether, the energy associated with which does not return to the radiator.

This conception leads to more correct ideas than are current on the mechanism of radiation from an antenna and permits explanation of the radiation from a coil aerial, which is not covered at all by the usual explanations of radiation in textbooks. Such explanations have led to the impression that the radiation largely depends on the form of the electrostatic lines of force which are present at the edges of the radiator. It might thus be supposed that in a flat-top antenna or a condenser aerial the current in the central portions of the condenser was not effective in causing radiation while only that which spread into the surrounding space from the edges was effective. This appears incorrect. If it were correct, the builders of long flat-top antennas must have wasted a great deal of wire. All of the dielectric current sends a moving disturbance out into the ether. The portion of the energy associated with this disturbance that does not return to the radiator is that connected with the first term of equation (3I). In this 
term the total antenna current appears. The radiation is the moving disturbance caused by the whole of the current which the antenna makes flow in the dielectric.

The ordinary treatment of the mechanism of radiation from an antenna is misleading also, because it deals with radiation at the fundamental wave length. In practice antennas are usually loaded. The radiation depends in no degree whatsoever on the value or location of any of the field lines attached to the aerial, but only on the variation of the lines. And all the lines when varying give rise to radiation. Thus the stationary field is given by the second term of formula (3I), the first is the radiation term, and they are independent.

\section{COMPARISON FORMULAS}

\section{DERIVATION FROM THEORETICAL FORMULAS}

Formulas are here derived to answer the practical question of how far a given coil will send or receive in comparison with a given antenna. The formulas also answer such questions as the length of a coil aerial required to give a particular ratio of performance of coil and antenna.

The ratio of the magnetic field radiated from a coil to that from an antenna, for a given sending current, distance, wave length, and height, is obtained from equation (13). The ratio of the distance from a coil to that from an antenna, at which a given magnetic field is produced, is the same as the ratio of the magnetic field produced by a coil to that produced by an antenna at a given distance. Either ratio is, therefore, given by the following expression, which assumes the same current $I_{8}$, wave length $\lambda$, and height $h_{8}$, for the coil and the antenna.

$$
\frac{d_{\mathrm{c}}}{d_{\mathrm{a}}}=N_{\mathrm{s}} \sqrt{2(\mathrm{I}-\cos \theta)}
$$

Inserting the value of $\theta$ and neglecting the subscript $\mathrm{s}$,

$$
\frac{d_{\mathrm{c}}}{d_{\mathrm{a}}}=N \sqrt{2\left(\mathrm{r}-\cos 2 \pi \frac{l}{\lambda}\right)}
$$

When the length of the coil $l$ is small compared to $\lambda$ (i. e., for most practical purposes, less than o.I $\lambda$ ), this simplifies to

$$
\frac{d_{\mathrm{o}}}{d_{\mathrm{a}}}=6.28 N \frac{l}{\lambda}
$$


This could have been deduced directly from (8) and (Io). The expression is similarly, deduced for comparison of the distances obtained with a coil and an antenna of different heights,

$$
\frac{d_{\mathrm{c}}}{d_{\mathrm{a}}}=6.28 N \frac{l}{\lambda} \frac{h_{\mathrm{o}}}{h_{\mathrm{a}}}
$$

The length of a coil required to give a particular ratio of performance to an antenna is given by solving these formulas for $l$. From (32),

$$
l=\frac{\lambda}{2 \pi} \cos ^{-1}\left(\mathrm{I}-\frac{\mathrm{I}}{2 N^{2}}\left[\frac{d_{\mathrm{c}}}{d_{\mathrm{a}}}\right]^{2}\right)
$$

When the length of the coil is small compared to $\lambda$, the simpler formula suffices,

$$
l=0.16 \frac{\lambda}{N} \frac{d_{\mathrm{o}}}{d_{\mathrm{a}}}
$$

The relative distances at which an antenna or a coil will receive a given wave are given by the same identical expressions that have just been deduced for sending aerials. Thus formula (32) may be deduced from (28) and (33) from (19) and (23). They give the ratio of the distance from the source at which a given emf will be produced in a coil aerial to that in an antenna, assuming the same height $h_{\mathbf{r}}$ and wave length $\lambda$ for the coil and the antenna. They also give the ratio of the emf produced in a coil to that in an antenna for a given value of magnetic field intensity, or the ratio of currents when the resistances and other quantities are the same in coil and antenna. Equations (34), (35), and (36) similarly hold for receiving as well as sending aerials. For comparison of current in a coil and an antenna of different resistances as well as different heights

$$
\frac{d_{\mathrm{c}}}{d_{\mathrm{a}}}=6.28 N \frac{l}{\lambda} \frac{h_{\mathrm{c}}}{h_{\mathrm{a}}} \frac{R_{\mathrm{a}}}{R_{\mathrm{c}}}
$$

The relative distances of transmission between two coil aerials and between two antennas, for a given sending current, is similarly found from equations (8), (10), (19), and (23). The ratio of received current for coils and antennas the same distance apart is given by the same formula, which assumes the same sending current $I_{\mathrm{s}}$ and wave length $\lambda$ for the pair of coils as for the pair of antennas.

$$
\frac{d_{\mathrm{cc}}}{d_{\mathrm{aa}}}=39.5 \frac{l_{\mathrm{s}} l_{\mathrm{r}} N_{\mathrm{s}} N_{\mathrm{r}}}{\lambda^{2}} \frac{\left(h_{\mathrm{s}} h_{\mathrm{r}}\right)_{\mathrm{c}}}{\left(h_{\mathrm{s}} h_{\mathrm{r}}\right)_{\mathrm{a}}} \frac{R_{\mathrm{a}}}{R_{\mathrm{o}}}
$$


All of these formulas assume that the decrement correction factor $F_{2}$ is the same for coil and antenna in all cases. If waves of differing decrement are used, apply the factor $F_{2}$ as stated in connection with (20). If the plane of the coil considered is not parallel to the direction of propagation of the wave, apply the factor, $\cos a$, as stated in connection with (24).

\section{EXAMPLES OF COMPARISON OF COIL AND ANTENNA}

What is the length of the coil, either as sender or receiver, equivalent to an antenna of the same height? The answer is given by $(36)$. For $\frac{d_{c}}{d_{a}}=\mathbf{I}$,

$$
l=0.160 \frac{\lambda}{N}
$$

This is the correct length except for a single-turn coil. When $N=$ I the more exact formula (35) must be used. This gives, for the equivalent coil,

$$
l=\frac{\mathrm{I}}{6} \lambda
$$

Thus a single-turn coil of length $1 / 6$ the wave length is equivalent to an antenna of the same height. For a coil of 8 turns, however, the length of the coil equivalent to an antenna of the same height is, from (39), 0.02 of the wave length.

When the length of the coil is small compared with the wave length - that is, as already stated, when $l$ is less than about o. I $\lambda$-the performance ratio is given by (33). For a length greater than O.I $\lambda$, however, the more accurate formula (32) must be used. Thus, when the length is exactly $1 / 6$ of a wave length, from (32),

$$
\frac{d_{\mathrm{c}}}{d_{\mathrm{a}}}=N
$$

Thus any coil of length. $1 / 6$ the wave length is equivalent to an antenna of $N$ times the height of the coil. When the length of the coil is one-quarter wave length, similarly

$$
\frac{d_{\mathrm{c}}}{d_{\mathrm{a}}}=\sqrt{2} \mathrm{~N}
$$

For a coil of length equal to one-half the wave length,

$$
\frac{d_{\mathrm{c}}}{d_{\mathrm{a}}}=2 N
$$


This is the maximum or best performance for a coil aerial. If the length is increased beyond one-half wave length, the performance ratio decreases, and at $l=\lambda$ it is equal to o just the same as for $l=0$.

These values of the performance ratio of a coil aerial are obvious from Fig. 5 or 6.

These comparisons all apply to either transmitting or receiving aerials. They assume, however, in the case of a transmitting antenna or coil, that the same current flows, and, when applied to receiving aerials, that the resistance is the same, in either coil or antenna. As a matter of fact, however, it is easy to secure a considerably lower resistance in a coil aerial circuit than in an antenna circuit. This is taken account of by the factor $\frac{R_{\mathrm{a}}}{R_{\mathrm{o}}}$ as in (37) and (38). The difference in current in a transmitting coil and antenna is taken account of by multiplying the right-hand members of $(32)$ and $(33)$ by the ratio of the sending currents $\frac{I_{c}}{I_{a}}$. On this account a coil is sometimes a more effective radiating or receiving device than an antenna of considerably greater dimensions.

The comparison formulas and conclusions drawn from them are subject to the same errors as the transmission formulas, as discussed in Section V, 2, below.

\section{THE CONDENSER AERIAL}

Since the dimensions of a coil aerial which would give the same performance as a given antenna are a length equal to $\frac{0.16}{N}$ times the wave length and a height equal to the antenna height, rather large structures are required. For example, a flat-top antenna $30 \mathrm{~m}$ above the ground operating on a $600 \mathrm{~m}$ wave is equivalent to a 4 -turn coil $24 \mathrm{~m}$ long by $30 \mathrm{~m}$ high. The dimensions of the equivalent coil are thus of the same order as the dimensions of the antenna.

It is possibie to escape from the apparent necessity of large structures for effective radio transmission and reception in two ways. First, the coil aerial can easily be made to have a lower resistance than the antennas ordinarily used, and its size reduced in proportion to the reduction of resistance. This is mainly because the condenser used in the coil aerial circuit can be one having 
practically no resistance while the condenser consisting of antenna and ground has a large resistance. Thus by due attention to the minimizing of resistance in its circuit, the coil aerial may be of small dimensions and yet highly effective. The size may, of course, be reduced also in proportion as the number of turns is increased.

It is equally possible to avoid an aerial of large dimensions without having recourse to a coil aerial. The alternative is to use the antenna principle, but use a special construction of much lower height. At first sight it would appear that this would make a poorer antenna, since the effectiveness is proportional to the height, according to either (8) or (I9). And this is true if the antenna is merely lowered a moderate amount. Such lowering increases the capacity only very slightly, not nearly in proportion to the decrease in height. In order to secure an appreciable gain it is necessary to have the height very small and use a special construction to reduce the resistance as much as possible. A good method is to replace the ordinary antenna-ground structure in which the antenna is one plate of a condenser and the ground the other plate, by an aerial consisting of two horizontal metal condenser plates. This may be called a "condenser aerial." The formulas derived for antennas apply to it.

Such an aerial has lower conductor resistance than the ordinary antenna, and since it has greater capacity a smaller inductance will be used in series with it which will also have smaller resistance and thus reduce the resistance of the circuit. Furthermore, the resistance of an antenna largely arises from the imperfect dielectrics, such as vegetation, buildings, and poor insulators, present in its field (as shown in Scientific Paper of the Bureau of Standards, No. 269 , by J. M. Miller), and the resistance from the grounding wires to ground. These can be eliminated in a condenser aerial. Finally, then, the resistance of the aerial circuit can be reduced to more than compensate for the reduction in height. This will result in a larger current $I_{\mathrm{r}}$ in formula (19), or in a larger $H$ in formula (8), because of the increase of the sending current.

The advantage of the very low antenna has been observed in the experiments of Kiebitz ${ }^{1}$ and others on so-called earth antennas. It is probable that still greater advantages would be obtained by the condenser aerial as here described. The special construction required to eliminate dielectric loss would involve making the lower plate considerably wider and longer than the 
upper plate, or else having both plates a considerable distance above the ground, and keeping the space between the plates free from poor dielectrics. An aerial consisting of a pair of metal plates elevated from the ground was used, described by Oliver Lodge in 1897 , and again by Lodge and Muirhead (Proc. Royal Soc., vol. 82 , p. 227 , 1909), who found that it worked best without being grounded. The author is informed that the same sort of an aerial has recently been tried on airplanes, using the upper and lower planes as the condenser plates. Such an aerial would be ideal for airplanes if the space between could be kept free from poor dielictrics. If the plates of the condenser aerial have their length and width approximately equal, the aerial radiates in all directions. If a long narrow condenser is used it would probably be very directional, both as a transmitting and receiving device. Such a condenser might consist of a pair of parallel wires, which would be a considerable improvement on the ground antenna.

An example will make clear how the size of the condenser aerial compares with other aerials. It was found above that an antenna $30 \mathrm{~m}$ high was equivalent to a 4 -turn coil $24 \mathrm{~m}$ long by $30 \mathrm{~m}$ high, both operating on a $600 \mathrm{~m}$ wave and with circuits of the same resistance. For the same wave length and with an inductance of 100 microhenries, in series, the capacity of a condenser aerial would need to be 0.00102 microfarad, which would be given by a pair of square plates $\mathrm{I} \mathrm{m}$ apart and $10.7 \mathrm{~m}$ on a side. The height is thus reduced in the ratio of about 25 to $\mathrm{r}$, and the horizontal dimensions 3 to $\mathrm{I}$ in comparison with the coil aerial.

The aerial can be made as small as desired. If a given coil is to be used in series, the capacity of the aerial is maintained constant by reducing the distance between the plates when the area of the plates is reduced. The author made some interesting experiments with a small condenser aerial as a receiving device, used inside the laboratory with no ground connection. The plates consisted of copper netting. The top plate was $250 \mathrm{~cm}$ square and the distance between them was $15 \mathrm{~cm}$. The signals received, with either a crystal detector or electron tube, were roughly of the same intensity as those received with a simple coil aerial of the type and size ordinarily used as a direction finder.

The indication of absolute direction of propagation of the waves as well as the line of propagation, which has been developed by workers in France and elsewheré, using combinations of ordinary 
antenna and coil aerials, was observed in the experiments on the condenser aerial. An inductance coil of rather large dimensions used in series with the condenser acted as a receiving aerial. As this coil was rotated the signal varied from maximum in one angular position to zero in a position $180^{\circ}$ from the first position; whereas the angular positions corresponding to maximum signal and to zero signal would be $90^{\circ}$ apart in the case of a coil aerial independent of any antenna action. Apparently the action of the condenser aerial reinforced that of the coil in one position and neutralized it in the opposite position. When the connections to the coil were interchanged, the effect shifted $180^{\circ}$. Reversing the connections of the coil reverses the emf in the coil, $E$, in Fig. 6, just as a reversal of the direction of the wave would do, whereas the direction of the emf in the antenna or condenser aerial is unchanged. The reason why the condenser emf can neutralize the coil emf is probably that the capacity of the coil introduces different values of reactance to the two emf's. Thus when the circuit is tuned for one of these emf's the currents due to the two differ $90^{\circ}$ in phase. This phase angle may be shifted $180^{\circ}$ by a very slight variation of the reactance of the circuit. Because of this, systems for determining the absolute direction of radio waves require very delicate adjustment.

The ordinary laboratory type of condenser used in radio circuits does not function as a condenser aerial. This is because the interleaving of the plates results in the current in each portion of the dielectric being balanced by the current in a neighboring portion. This is discussed further below in Section VI, 3, and illustrated in Fig. I 7 . 


\section{TRANSMISSION FORMULAS}

\section{STATEMENT OF FORMULAS}

The current received in any aerial may be calculated in terms of the current in any transmitting aerial, either antenna or coil, by the following four formulas. They are derived by combining equations (8), (I0), (I9), and (23). The symbols are as previously given, also stated in the Appendix below.

Antenna to antenna:

$$
I_{\mathbf{r}}=\frac{188 . \quad h_{\mathrm{s}} h_{\mathrm{r}} I_{\mathrm{s}}}{R \lambda d}
$$

Antenna to coil:

$$
I_{\mathrm{r}}=\frac{\mathrm{II} 84 \cdot h_{\mathrm{s}} h_{\mathrm{r}} l_{\mathrm{r}} N_{\mathrm{r}} I_{\mathrm{s}}}{R \lambda^{2} d}
$$

Coil to antenna:

$$
I_{\mathrm{r}}=\frac{\mathrm{II} 84 \cdot h_{\mathrm{s}} l_{\mathrm{s}} h_{\mathrm{r}} N_{\mathrm{s}} I_{\mathrm{s}}}{R \lambda^{2} d}
$$

Coil to coil:

$$
I_{\mathrm{r}}=\frac{7450 . \quad h_{\mathrm{s}} l_{\mathrm{B}} h_{\mathrm{r}} l_{\mathrm{r}} N_{\mathrm{s}} N_{\mathrm{r}} I_{\mathrm{s}}}{R \lambda^{3} d}
$$

The formula (40) has existed heretofore in various forms. The formulas here given generalize the antenna-to-antenna formula, so that calculations can be made for any kinds of aerials.

The lengths in these formulas may be in any units, provided the same unit is used for all the lengths. The meter is usually the most convenient unit. If the heights and wave length are in meters and the distance $d$ in miles, the four constants in the four formulas become, respectively, $0.117,0.736,0.736$, and 4.63 .

To calculate the distance at which a given current will be received, as when a particular receiving arrangement is specified, the formulas may be stated explicitly for $d$. $I_{\mathbf{r}}$ and $d$ are interchanged in each formula. For example, the formula for antennato-coil (4I) becomes

$$
d=\frac{\mathrm{II} 84 \cdot h_{\mathrm{s}} h_{\mathrm{r}} l_{\mathrm{r}} N_{\mathrm{r}} I_{\mathrm{s}}}{R \lambda^{2} I_{\mathrm{r}}}
$$


All of these transmission formulas are for daytime transmission. Greater values are obtained at night, probably because the waves are reinforced by reflection from ionized layers of the upper atmosphere, which are broken up by sunlight in the daytime. The formulas are all subject to correction factors for distance and for decrement. If the distance is very great (in ordinary cases, over roo kilometers), the right-hand side of the formula should be multiplied by the correction factor $F_{1}$. The value given below for $F_{1}$ is for transmission over sea water. Its value for transmission over land would be greater. If damped waves are used, the correction factor $F_{2}$ should be similarly applied. Futhermore, if the plane of the receiving coil is not parallel to the direction of propagation of the wave, the correction factor $F_{3}$ must be similarly applied to formulas (4I) and (43) and related formulas, such as (44). In formulas (42) and (43) the direction of the wave is taken to be that of the plane of the transmitting coil. The three correction factors are:

$$
\begin{gathered}
F_{1}=\epsilon^{-0.000047 \frac{d}{\sqrt{\lambda}}} \\
F_{2}=\sqrt{\frac{\mathrm{I}}{\mathrm{I}+\frac{600 \cdot L \delta^{\prime}}{R \lambda}}} \\
F_{3}=\cos a
\end{gathered}
$$

All of the correction factors make the resultant numerical values smaller.

\section{DISCUSSION OF TRANSMISSION FORMULAS}

The power in which the wave length appears in the denominator is different in the several formulas. Thus when a coil aerial is used for both transmitter and receiver the received current is inversely proportional to the cube of the wave length. Thus transmission between coils is better the shorter the wave length. This advantage of coils at short wave lengths applies only for short-distance transmission. When the distance is hundreds or thousands of kilometers, the increased absorption of the waves makes the correction factor $F_{1}$ so great that short waves are impractical. So for long distances the comparison favors the antenna rather than the coil. The coil compares most favorably with the antenna, then, for transmission over short distances with very short waves. This is subject to the proviso that current of the same order of 
magnitude can be put into a transmitting coil aerial as into an antenna, or that the resistance of a receiving coil is the same as that of a receiving antenna. Neither of these assumptions is wholly fulfilled, in practice, with the result that the difference of applicability of the two kinds of aerials at long and short wave lengths is less marked. (For additional comparisons of antennas and coils and further discussion, see Section IV above.)

Limitations of Formulas.-The formulas can not be expected to give results of great accuracy, certainly not better than a few per cent, because of the ideal conditions assumed in their derivation. Thus it is assumed that no image of the aerial exists in the ground beneath it; that is, the ground is not perfect as a conductor. As a matter of fact, the ground varies greatly in conductivity, and while in most cases the currents induced in the ground below a transmitting or receiving aerial probably have very little effect, these currents may be appreciable in some cases. This is discussed further below under "Height of Aerial."

On account of the uncertainty introduced by the ground, the formulas may apply better to airplane aerials than to those on ships or on land. It is well known, however, that radio signals are fainter on an airplane than on the ground. It is not known to what extent this is due to $(I)$ the diminution of intensity vertically of waves sliding along the ground, (2) electrical disturbances from the ignition system of the engine, and (3) the unavoidable noises on an airplane.

In transmission over great distances, particularly at night, radio waves are reinforced by reflection from the upper conducting strata of the atmosphere. This tends to increase the current in a receiving aerial. The theory of this effect is treated in an article by $\mathrm{G} . \mathrm{N}$. Watson (Proc. Royal Soc., vol. 95, p. 546; 1919).

There are other sources of uncertainty in the application of these formulas. An antenna does not form a flat-plate condenser with the ground of such form that the curving of the field at the edges can be neglected. The simple method of calculating the radiated field is thus in doubt. Similarly, in the case of a radiating coil, the field from the top and bottom of the coil may have some effect at a distance, which has not here been taken into account. It is not certain with how great propriety the earth's surface can be taken to be equivalent to the equatorial plane of the radiating aerial. Frequently radio waves have a wave front that is tilted and not perpendicular to the earth's surface, as 
assumed in the calculation of received current. Furthermore, the formulas assumed uniform current throughout the aerial, which sometimes does not hold, because the antenna may have a vertical portion of appreciable capacity or the coil may have rather large distributed capacity. Calculations involving coil aerials are subject to the additional uncertainty arising from the capacity of the coil to ground or the surroundings so that it acts like an antenna as well as a coil. This is discussed under "Antenna Effect" in Section VI, 3. Another difficulty discussed in the same section is the effects of surrounding objects.

With these departures in the action of the aerials and the behavior of the waves from the conditions assumed, it is impossible to calculate received currents with great accuracy. It is almost surprising that the experimental results check the formulas as closely as shown in Section VI, 2, below.

Height of Aerial.-The value used for $h$ is the length of the vertical side of a coil aerial, the distance from the surface of the ground to the flat top of an antenna, or the vertical distance between the two flat plates of a condenser aerial. In previous discussions it has been assumed that the ground beneath an antenna was a perfect conductor and thus the height of the radiator was twice the value of the $h$ defined here. Experiment, however, corroborates the view here taken, which assumes that the radiating structure is independent of the earth, the waves becoming attached to the earth soon after leaving the antenna. In the present state of our knowledge the most satisfactory conception is that the radiating structure is the actual structure above the ground level. (Questions of the conductivity of the ground, presence of earth currents, etc., near the radiating aerial, are expressly not considered.)

Austin's empirical formula ${ }^{2}$ for antenna-to-antenna transmission is equation (40) with a constant twice as great, and quantities $h_{1}$ and $h_{2}$ used instead of $h_{\mathrm{s}}$ and $h_{\mathrm{r}}$. These quantities $h_{1}$ and $h_{2}$ are the "height to the center of capacity" of the transmitting and receiving antenna, respectively. This height is not defined, but its value for any particular antenna is the value that is required to make experimental results fit the formula. Now, as has been stated, such experiments as have been performed agree in general with formula (40). For instance, see the first two examples in Section VI, 2, below. It must follow, since the 
constant in Austin's formula is twice as great as the constant in (40), that

$$
h_{1} h_{2}=\frac{\mathrm{I}}{2} h_{\mathrm{s}} h_{\mathrm{r}}
$$

This may be satisfied by various values of $h_{1}$ and $h_{2}$. One set of values would be

Another would be

$$
\begin{aligned}
& h_{1}=0.5 h_{\mathrm{s}} \\
& h_{2}=1.0 h_{\mathrm{r}}
\end{aligned}
$$

$$
\begin{aligned}
& h_{1}=0.707 h_{\mathbf{s}} \\
& h_{2}=0.707 h_{\mathbf{r}}
\end{aligned}
$$

Austin's values for the height of various antennas, thus deduced in such a way as to make them fit the experimental values observed, do in fact vary from one-half to full value of the actual antenna heights, and average around 0.7 the actual heights. It is much simpler and more direct to use the formula and the interpretation presented in this paper, bearing in mind that it is subject to the uncertainties introduced by the varying character of the ground.

The idea that the ground is not a good enough conductor to form an image of a transmitting aerial, and that the waves become attached to the ground after leaving the aerial, is in harmony with the ideas of Lodge and Muirhead, already referred to. They found that they got better transmission by using what amounted to a condenser aerial, elevated from ground, with no ground connection. This conception conflicts with the commonly accepted view that Marconi's achievement was the connection of a radiating system to the ground. What then was Marconi's achievement? The best answer to this may be one stated to the author by Prof. A. E. Kennelly, viz, the use of a large radiating system, arranged vertically.

\section{EXPERIMENTAL VERIFICATION OF FORMULAS}

\section{PRINCIPLES OF MEASUREMENT OF RECEIVED CURRENT AND VOLTAGE, WITH APPLICATIONS TO DESIGN}

The formulas presented in this paper not only make it possible to calculate approximately the field intensity produced or current received with given aerials, but also give the basis for determining what constants to select for the circuit of a particular aerial to secure the maximum effect. In other words, these formulas fur- 
nish the principles of design of aerial circuits. There are a great many points not obvious from mere inspection of the formulas, which are of importance equally in design and in the measurement of received signals. These will now be considered. While this discussion is limited to what takes place in receiving aerials, the same principles and treatment can readily be applied to transmitting aerials.

The received current or voltage can be measured in a number of different ways. It is important to know just what quantity is being considered or measured. Suppose an indicating instrument $G$, which may be a galvanometer or a telephone receiver, is connected to a rectifying device $D$ in parallel with the condenser of the receiving circuit, as in Fig. 7 , where either $L$ is a coil aerial,

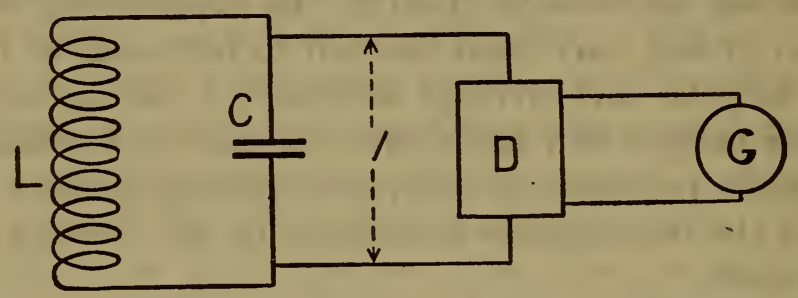

FIG. 7.-Aerial circuit with detecting apparatus across the condenser

or else $C$ is an antenna or condenser aerial. Does the indication of the instrument measure directly $(a)$ the emf which the wave causes to act on the circuit $C L,(b)$ the current in the circuit, or (c) the voltage across the condenser? The answer is, of course, none of these things. The system can, however, conveniently be calibrated in terms of the voltage across the condenser. This voltage $V$ is related to the received current $I_{\mathbf{r}}$ by the relation

$$
V=\frac{I_{\mathrm{r}}}{\omega C}
$$

and since $I_{\mathrm{r}}$ is related to the emf acting by

$$
I_{\mathrm{r}}=\frac{E}{R}
$$

the relation of $V$ to $E$ is

$$
V=\frac{E}{R \omega C}
$$


When a detecting apparatus like that of Fig. 7 is used, in which the deflections or signals depend directly on the voltage across the condenser, the results obtained with various receiving circuits will be entirely different from those obtained when the current in the circuit is directly measured, as in Fig. 8. Equations (46) and (47) show at once that the effects of varying the constants of the receiving circuit will be different, depending on whether it is $E$, $I_{r}$, or $V$ that is being measured. These three quantities for a receiving antenna are, from equations ( 17$),(46)$, and (47), for unit magnetic field intensity,

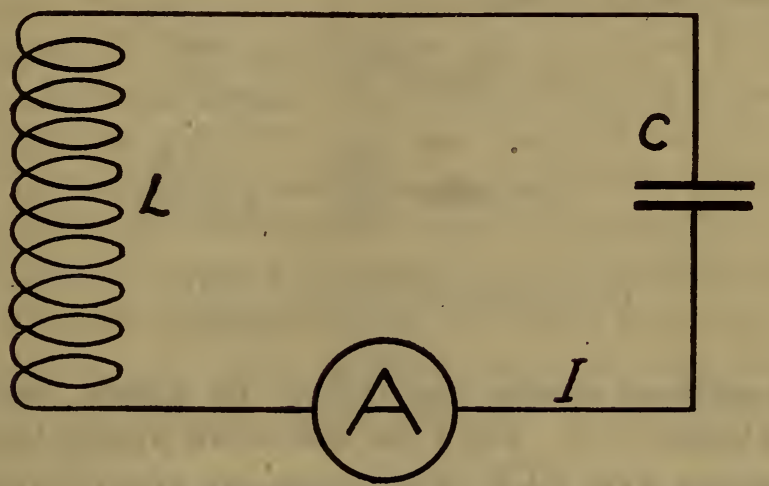

FIG. 8.-Aerial circuit with current-measuring instrument in circuit

$$
\begin{aligned}
E_{\mathrm{a}} & =300 \cdot h_{\mathrm{r}} \\
I_{\mathrm{a}} & =300 \frac{h_{\mathrm{r}}}{R} \\
V_{\mathrm{a}} & =300 \frac{h_{\mathrm{r}}}{R \omega C}
\end{aligned}
$$

The quantity $h_{\mathrm{r}}$ may be called the "emf reception factor" for an antenna; the emf in the receiving circuit is proportional to it. Similarly $\frac{h_{\mathrm{r}}}{R}$ may be called the "current reception factor" since it determines the received current. And $\frac{h_{\mathrm{r}}}{R \omega C}$ or the equivalent $\frac{h_{\mathrm{r}} L}{R \lambda}$ may be called the "voltage reception factor" of an antenna since it determines the voltage across the antenna.

The most favorable or optimum value of any of the variables that determine the antenna emf, current, or voltage, can be determined either by direct experimental measurement of their values when actually receiving or by calculation from the reception 
factors. It is desired to learn simply what will produce the maximum $E_{\mathrm{a}}, I_{\mathrm{a}}$, or $V_{\mathrm{a}}$. For example, it is obvious that $E_{\mathrm{a}}$ increases indefinitely as $h_{\mathrm{r}}$ increases, but more careful consideration is required to determine what will be the effect on the received current of increasing $h_{\mathrm{r}}$. The reception factors furnish an alternative to direct reception measurements, requiring instead measurements upon the constants of the aerial circuit.

Coil Aerial Reception Factors.-The emf applied by the passing wave to a coil aerial, the current in the circuit, and the voltage across the condenser are, from equations (26), (46), and (47), for unit magnetic field intensity,

$$
\begin{gathered}
E=600 . \pi \frac{a^{2} N}{\lambda}=\frac{\mathrm{I}}{\mathrm{IO}} \frac{a^{2} N}{\sqrt{C L}} \\
I=600 . \pi \frac{a^{2} N}{R \lambda}=\frac{\mathrm{I}}{\mathrm{IO}} \frac{a^{2} N}{R \sqrt{C L}} \\
V=36 . \pi^{2} \mathrm{IO}^{12} \frac{a^{2} N L}{R \lambda^{2}}=\frac{\mathrm{I}}{\mathrm{IO}^{8}} \frac{a^{2} N}{R C}
\end{gathered}
$$

These equations assume the coil to be square, having both height and length $=a$. For a coil that is not square, the formulas apply, replacing $a$ by $\sqrt{h_{\mathrm{r}} l}$. Two values are given for each reception factor; the first of the two is the more useful, since it is more common to consider the dependence of the reception on $\lambda$ than on $C$.

$$
\begin{gathered}
\text { Emf reception factor }=\frac{a^{2} N}{\lambda} \\
\text { Current reception factor }=\frac{a^{2} N}{R \lambda} \\
\text { Voltage reception factor }=\frac{a^{2} N L}{R \lambda^{2}}
\end{gathered}
$$

Design of Receiving Coil Aerials.-The response produced in a coil aerial circuit may be measured in a great variety of ways. In the first place, it may be considered either from the viewpoint of the emf acting, the current, or the voltage across the condenser. The first of these, the emf $E$, may be determined for any particular case from the emf reception factor (5I). The current $I$ or voltage $V$ may each be determined in four different ways: (I) by direct measurement with a suitable instrument, (2) by measurement of the quantities which make up the appropriate reception factor, (3) by measurement of signal strength in some such device as 
sketched in Fig. 7, which has been calibrated in $I$ or $V,(4)$ from a "signal intensity reception factor," which can be calculated for any signal-measuring device when the law connecting the signal intensity with either $I$ or $V$ is known.

The design of a receiving coil requires knowledge of the dependence of the current or voltage upon the dimensions, etc., of the coil. Measurements made in all of the ways just enumerated give results in agreement with one another, provided due care is given to the avoidance of errors. The sources of error are numerous, as discussed in Section 3 below.

While direct measurement of the received current or voltage can be replaced by calculation from the reception factors, the fact remains that the design of an aerial requires experiments. This is because the quantity $R$ in the reception factors can not be obtained by calculation. It must be obtained by measurement for the particular coil and mode of connections employed.

Measurements upon receiving aerials to determine their constants and the best design for given conditions constitute a most interesting study. In later publications from this laboratory the results of experiments will be published giving such data for typical coil aerials.

The capacity $C$ in the formulas is the total capacity of the circuit, including the capacity of the coil, $L$ is the pure inductance of the coil, and $R$ is the actual resistance of the circuit. $R$ includes the resistance of the conductors, effective resistance of the condenser and of the coil capacity, effective resistance of the detecting apparatus, and radiation resistance. All of these vary with frequency, and thus measurements of $R$ at the frequency concerned is necessary. On account of the complexity of the quantities entering into the total $R$, its measurement is no easy matter. The capacity of the coil and other stray capacities may easily vitiate the measurement of $R, C$, or $L$. The effect of the detecting apparatus always requires most careful consideration. Even if $D$ in Fig. 7 is an electron tube, it is necessary to consider the resistance which it introduces into the aerial circuit.

Dependence of Received Current and Voltage on Dimensions of Coil and Wave Length.-Let $R_{\mathrm{c}}=$ resistance of coil and $R_{\mathbf{x}}=$ resistance external to coil;

$$
R=R_{\mathrm{x}}+R_{\mathrm{c}}
$$

$\mathrm{I} \propto$ Current reception factor $=\frac{a^{2} N}{\left(R_{\mathrm{x}}+R_{\mathrm{c}}\right) \lambda}$ 
The variation of received current with number of turns, wave length, and size of coil is readily found by considering the variation of the quantities in (54). In the following discussions the spacing between turns of wire, which affects resistance and inductance, is assumed constant.

(a) Varying $N$, with $\lambda$ and $a$ constant. When $R_{\mathbf{x}}$ is large compared to $R_{\mathrm{c}}$, we see from (54)

$$
I \propto N
$$

When $R_{\mathrm{c}}$ is large compared to $R_{\mathrm{x}}$, since $R_{\mathrm{c}} \propto N$, roughly, $I \propto$ constant

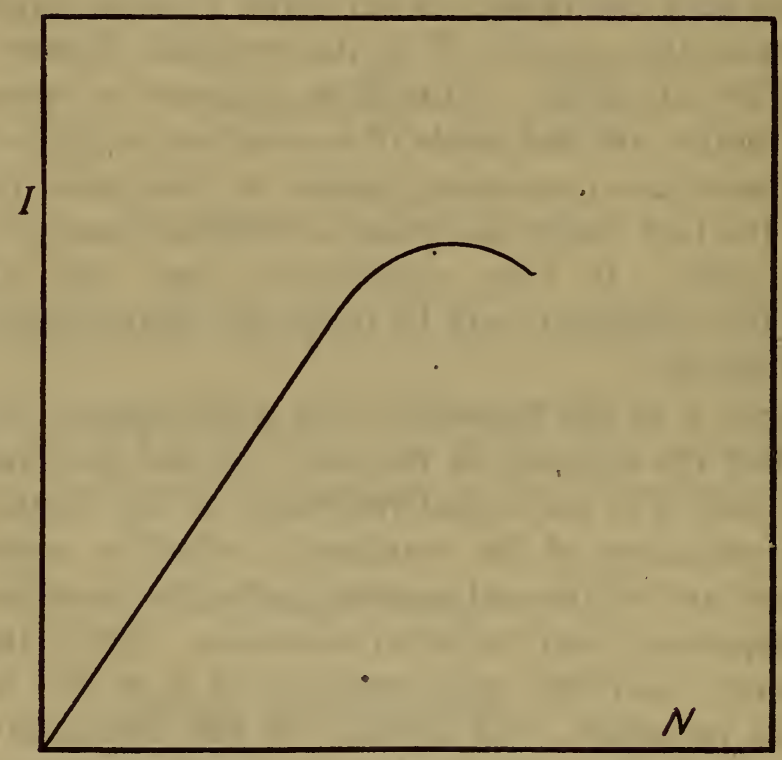

Fig. 9.-Dependence of received current on number of turns

However $R_{\mathrm{c}}$ increases somewhat faster than proportional to $N$ as $N$ is increased, because of the proximity of the added turns, and hence $I$ decreases somewhat as $N$ increases instead of being strictly constant.

$R_{\mathrm{x}}$ is likely to be large compared with $R_{\mathrm{c}}$ when $N$ is very small, and hence for small $N$, the variation of $I$ with $N$ will be a straight line, as shown in Fig. 9. As $N$ increases, $R_{\mathrm{c}}$ becomes large compared to $R_{\mathbf{x}}$ and the tendency of $I$ to increase with $N$ is reversed. As a result the curve of $I$ has a maximum. The value of $N$ at this point may be called the "optimum number of turns." Its value will be greater the greater the external resistance. 
(b) Varying $\lambda$ with $N$ and $a$ constant. When $R_{\mathrm{x}}$ is large compared to $R_{\mathrm{c}}$ and does not vary appreciably with wave length

$$
I \propto \frac{I}{\lambda}
$$

This variation is shown in Fig. Io. When $R_{\mathrm{c}}$ is large compared to $\dot{R}_{\mathbf{x}}$, however, since $R_{\mathrm{c}} \propto \frac{1}{\sqrt{\lambda}}$, roughly,

$$
\text { - } I \propto \frac{\mathrm{I}}{\sqrt{\lambda}}
$$

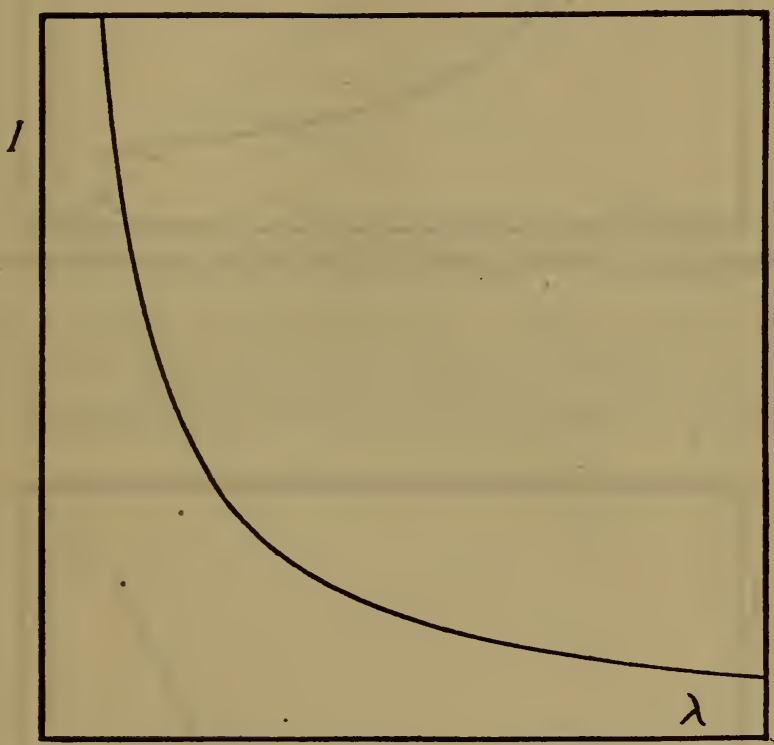

FIG. 10.-Dependence of received current on wave length when external resistance is large

However the effect of the adjacent turns increases $R_{\mathrm{c}}$ faster than stated, as $\lambda$ is diminished, and hence $I$ tends to approach a constant value for short wave lengths, as shown in Fig. II. These conclusions may, however, be vitiated by the variation of $R_{\mathbf{x}}$ with $\lambda$.

(c) Varying $a$, with $N$ and $\lambda$ constant. When $R_{\mathbf{x}}$ is large compared to $R_{\mathrm{c}}$,

$$
I \propto a^{2}
$$

This is shown in Fig, 12. When $R_{\mathrm{c}}$ is large compared to $R_{\mathbf{x}}$, since $R_{\mathrm{c}} \propto a$,

$$
I \propto a,
$$




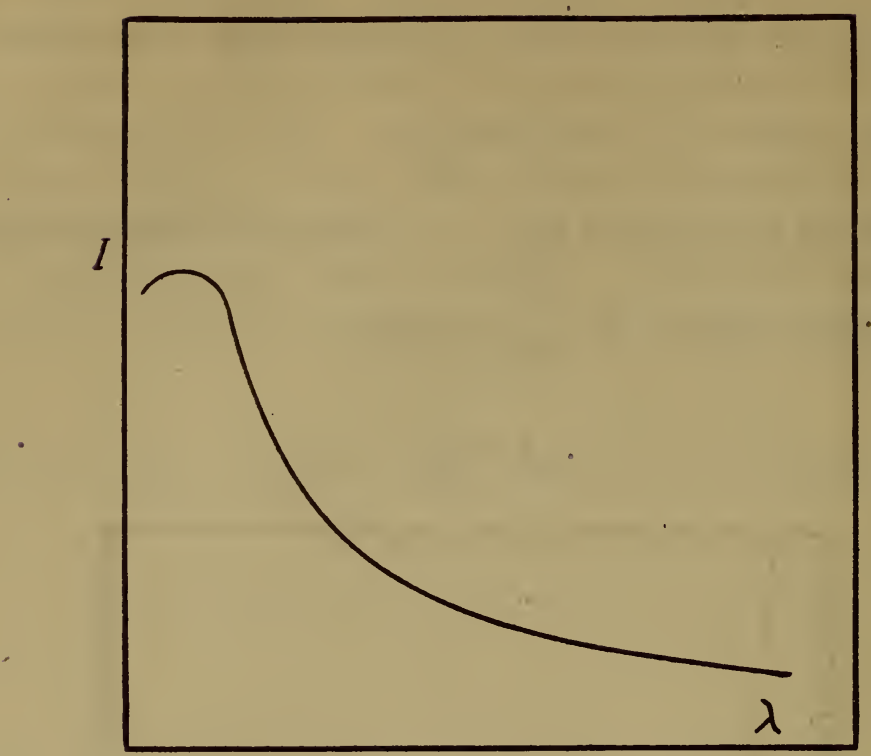

FrG. II.-Dependence of received current on wave length when coil resistance is large

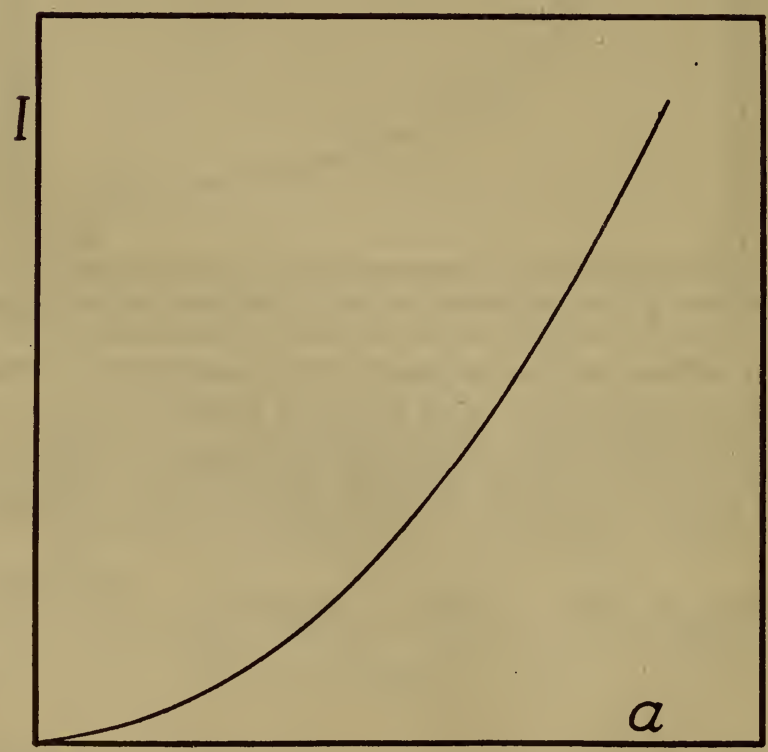

FIG. 12.-Dependence of received current on size of coil when external resistance is large 


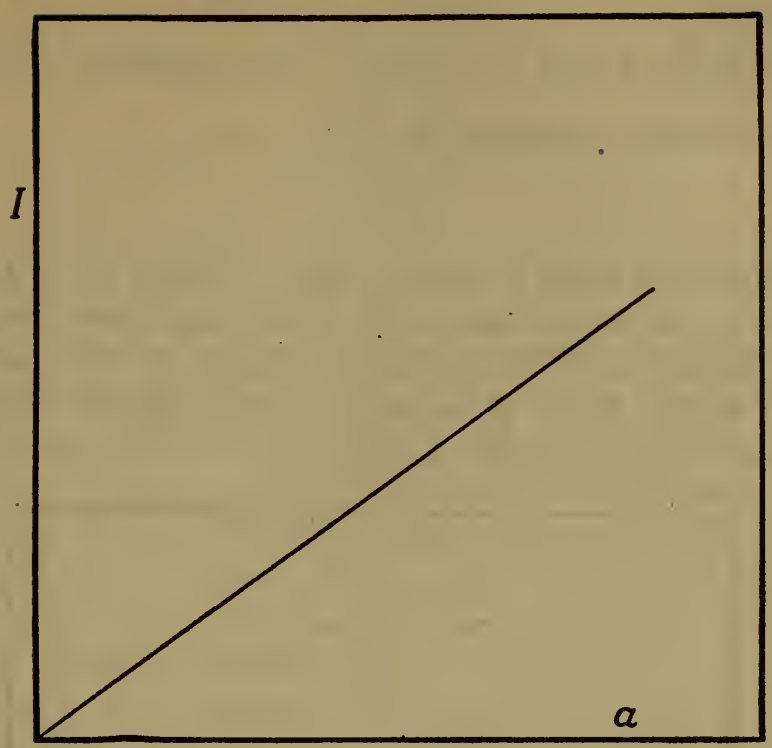

FIG. 13.-Dependence of received current on size of coil when coil resistance is large giving the straight line in Fig. 13. From these two extreme cases it follows that an actual curve is likely to have a form that is a combination of these two, as shown in Fig. I4.

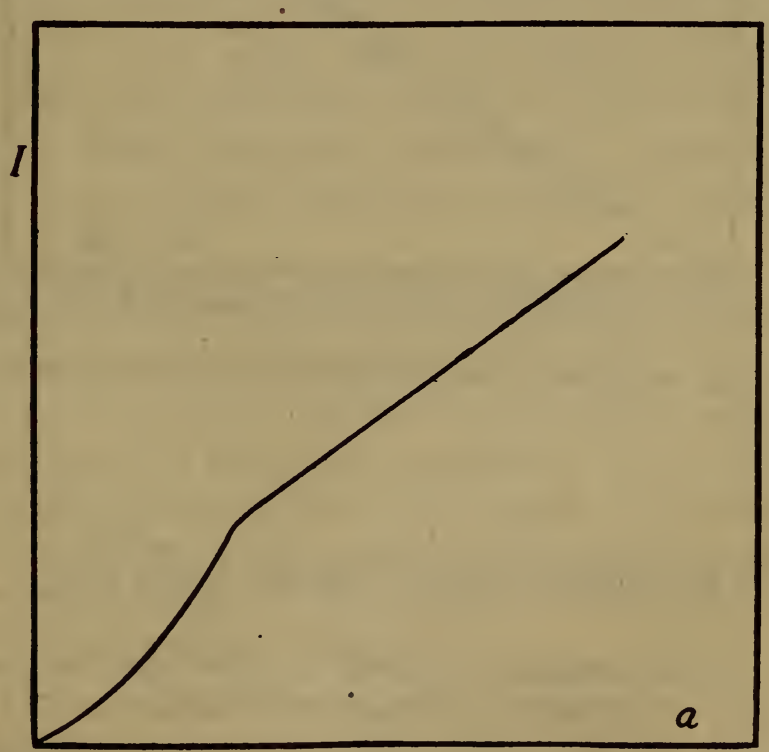

FIG. 14.-Dependence of received current on size of coil in typical case 
(d) Varying $a$, with $\lambda$ constant, allowing $N$ to vary in such a way that length of wire is constant. The condition is that $N \propto \frac{1}{a}$. When $R_{\mathrm{x}}$ is large compared to $R_{\mathrm{c}}$,

$$
I \propto a .
$$

The curve of $I$ is thus a straight line. When $R_{\mathrm{c}}$ is large compared to $R_{\mathrm{x}}$, the same conclusion holds, but only roughly. $R_{\mathrm{c}}$ increases slightly as $a$ is decreased because of the proximity of the added turns, hence $I$ increases a little faster than proportional to $a$. This is shown in Fig. I5.

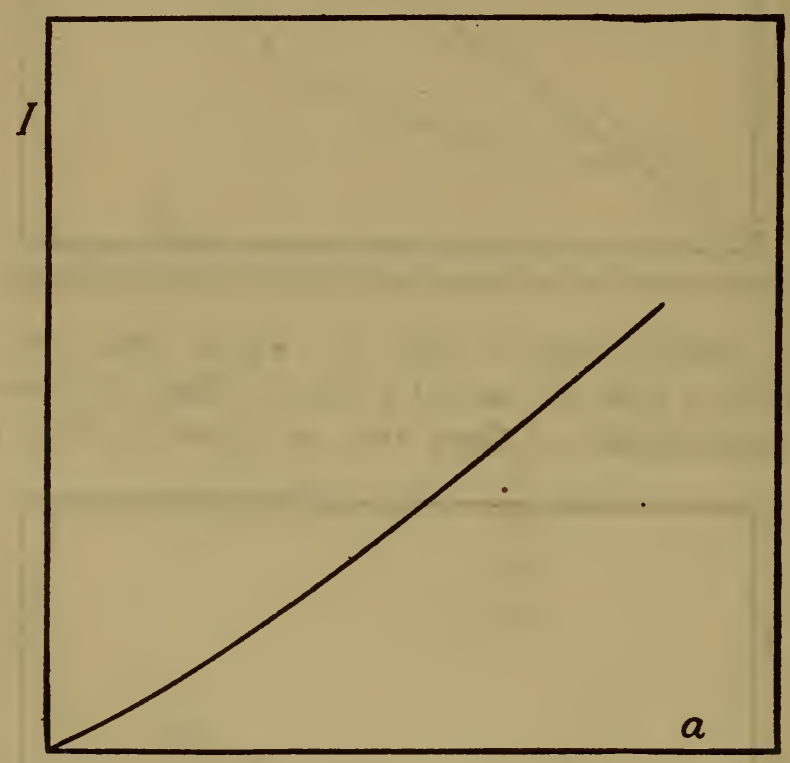

FIG. 15.-Dependence of received current on size of coil when length of wire is kept constant

The voltage reception factor differs from the current reception factor by $\frac{L}{\lambda}$.

Thus,

$$
V \propto \text { Voltage reception factor }=\frac{a^{2} N L}{\left(R_{\mathrm{x}}+R_{\mathrm{c}}\right) \lambda^{2}}
$$

It is thus a more complex problem to determine the variation of voltage with $N, \lambda$, and $a$, because the variation of $L$ must be considered in addition. This may be done in each case, just as was done above for current, taking into account the relations: 


$$
\begin{aligned}
& L \propto N^{2-} \\
& L \propto \lambda^{0-} \\
& L \propto a^{1+} \\
& R_{\mathrm{o}} \propto N^{1+} \\
& R_{\mathrm{o}} \propto \frac{\mathrm{I}}{\lambda^{3+}} \\
& R_{\mathrm{o}} \propto a^{1}
\end{aligned}
$$

where the + or - sign after the exponent indicates that the actual power is slightly greater or less than that given.

The relations obtained for voltage are similar to those obtained for current. There are some characteristic differences, as, e. g., with varying $N$ the optimum number of turns comes out greater than for received current. Thus, when the detecting apparatus depends essentially on the current, the size of the coil should be as large as possible, whereas when it depends essentially on the voltage across the condenser the number of turns should be as large as possible.

On the whole, the received current or voltage or signal intensity is increased by increasing the dimensions ( $N$ and $a$ ) and by decreasing the wave length. These conclusions are subject to the limitation, discovered by French experimenters, and qualitatively obvious from the known increase of $R$ near the natural wave length $\lambda_{0}$ of a circuit, that poor results are obtained if $\lambda_{0}>1 / 3 \lambda$. Thus, the dimensions of the coil can not be increased, or the wave length decreased, indefinitely. Beyond the limit mentioned, in fact, better results are obtained by decreasing the dimensions of the coil.

It is an interesting fact that these discussions apply not only to the design of a coil aerial for receiving signals, but that they also solve the problem of design of wave meters. The choice of constants of a wave-meter coil for any particular case is settled by the formulas and ideas here presented. The considerations given for received current and voltage apply, respectively, to the design of wave meters for measurements upon undamped or damped waves; that is, to the securing of minimum resistance and minimum decrement, respectively.

\section{EXAMPLES OF MEASUREMENTS}

Measurements having as their object the verification of the transmission formulas were discussed in the preceding section. Any experiments which verify the transmission formulas may also be considered as checking the "theoretical formulas" and 
"comparison formulas." In fact experimental tests of the transmission formulas are the most rigorous test of the theory presented in this paper. All of the limitations and errors discussed in Section V, 2, affect the transmission formulas, while only a portion of them affect the theoretical and comparison formulas.

The complicated practical conditions of any experiment, the tilting of the wave front, the combination of antenna and coil effects, discussed in the next section below, and other uncertainties, make close agreement between theory and experiment unlikely. Agreement to 30 per cent should be considered as highly satisfactory verification of the essential correctness of the theory. On account of its field being more definitely localized, experiments with a coil aerial may be expected to yield greater accuracy than experiments with an antenna. The same advantage appertains to a condenser aerial. No quantitative data have been obtained with condenser aerials, to the author's knowledge; such experimtents would be very desirable.

Experimental data obtained at the Bureau of Standards on radio transmission and reception are presented below. The agreement between the received current observed and the values calculated from the transmission formulas can be considered as very satisfactory. The author is informed that experiments made by the Signal Corps have led to a similar verification. In some of the Signal Corps experiments it was thought at one time that wide departures from the transmission formulas for coil aerials were observed, the received current for very short waves being much less than the transmission formulas indicated. When, however, the actual values of the resistance at the wave lengths used were determined, the agreement was very good. Particular care must be given to proper measurement of the resistance of the receiving aerial circuit.

Antenna to Antenna.-Experiments which supply a check on formula (40) have been published by Dr. Austin, chief of the Naval Radio telegraphic laboratory located at the Bureau of Standards.

For transmission between two antennas located on ships (Jour. Wash. Acad. Sciences, vol. I, p. 275; I9I I), $h_{\mathrm{s}}=29.2$, $h_{\mathrm{r}}=29.2, I_{\mathrm{s}}=30 ., R=25 ., \lambda=1000 ., d=1000$. The lengths given in all these examples are in meters. Calculating from (40),

$I_{\mathrm{r}}$ calculated $=0.19$

$I_{\mathrm{r}}$ observed $=0.2 \mathrm{I}$ 
For the Washington Navy Yard antenna transmitting to an antenna at the Bureau of Standards (Bull. Bureau of Standards, vol. I I, p. 74 ; 1914), $h_{\mathrm{s}}=36 ., h_{\mathrm{r}}=30 ., I_{\mathrm{s}}=7.0, R=70 ., \lambda=2800$., $d=$ I0 000. Calculating from (40) and (20)

$$
\begin{aligned}
& I_{\mathrm{r}} \text { calculated }=0.53 \times 10^{-3} \\
& I_{\mathrm{r}} \text { observed }=0.55-\mathrm{ro}^{-3}
\end{aligned}
$$

As already pointed out, the agreement of observed values with the transmission formulas indicates that it is proper to take as the antenna height the actual height from the ground to the flat top.

Antenna to Coil.-A number of experiments to check the antenna-to-coil transmission formulas have been made at the Bureau of Standards radio laboratory since early in I9I7. The first ones did not involve quantitative measurements, but served to give a rough check on the formulas. The calculated value of current was compared with the current as estimated from the loudness of signal in a telephone receiver connected to various types of detecting devices. These signals were interpreted on the assumption that a fairly audible response is given by the currents indicated with the several types of detector.

$$
\begin{aligned}
& \mathrm{IO}^{-4} \text { to } \mathrm{IO}^{-5} \text { ampere, crystal detector. } \\
& \mathrm{IO}^{-5} \text { to } \mathrm{IO}^{-6} \text { ampere, simple audion. } \\
& \mathrm{IO}^{-8} \text { ampere, oscillating audion. }
\end{aligned}
$$

For the Arlington antenna received on a coil aerial at the Bureau of Standards, $h_{\mathrm{s}}=122 ., h_{\mathrm{r}}=4 ., l_{\mathrm{r}}=4 ., N_{\mathrm{r}}=22 ., I_{\mathrm{B}}=102$., $R=25 ., \quad \lambda=3800 ., \quad d=7800$. The received current calculated from (4I) is 0.0018 ampere. The observed signal using crystal detector and telephone, was very loud, thus checking in a qualitative way the result calculated.

Two similar qualitative experiments were made, transmitting from an antenna at the Bureau of Standards and receiving on a portable coil aerial. In one experiment, $h_{\mathrm{s}}=36 ., h_{\mathrm{r}}=l_{\mathrm{r}}=1.07$, $N_{\mathrm{r}}=\mathrm{II}$., $I_{\mathrm{B}}=0.5, R=2 ., \lambda=850 ., d=\mathrm{I} 6000$., whence calculated $I_{\mathrm{r}}=11 . \times 10^{-6}$. In the other, $h_{\mathrm{s}}=12$., $h_{\mathrm{r}}=l_{\mathrm{r}}=1.07, N_{\mathrm{r}}=11 ., I_{\mathrm{s}}=0.25$, $R=2, \lambda=600 ., d=11000$, whence calculated $I_{\mathrm{r}}=5.7 \times 10^{-8}$. In both cases the observed signal was loud with a simple audion, again giving a qualitative check on the formula. 
A number of experiments have been made by Dr. Austin during 1918 and 1919, who has kindly placed the results at my disposal. A couple of typical ones will be given. For the Arlington antenna transmitting to a coil aerial at the Bureau of Standards, $h_{\mathrm{s}}=\mathbf{1 2 2}$., $h_{\mathrm{r}}=\mathrm{I} .82, l_{\mathrm{r}}=\mathrm{I} .29, N_{\mathrm{r}}=56 ., I_{\mathrm{s}}=100 ., R=50 ., \lambda=6000, d=7800$.

$$
\begin{aligned}
& I_{\mathrm{r}} \text { calculated }=\mathrm{I} .4 \times 10^{-4} \\
& I_{\mathrm{r}} \text { observed }=2.1 \times 10^{-4}
\end{aligned}
$$

For the same antenna transmitting to a large coil suspended from masts outdoors, $h_{\mathrm{s}}=122 ., h_{\mathrm{r}}=2 \mathrm{I} .6, h_{\mathrm{r}}=24.4, N_{\mathrm{r}}=7 ., I_{\mathrm{s}}=100$., $R=50$., $\lambda=$ I0 000, $d=7800, a=42^{\circ}$. From (4I) and (24),

$$
\begin{aligned}
& I_{\mathrm{r}} \text { calculated }=\mathrm{I} .0 \times 1 \mathrm{O}^{-3} \\
& I_{\mathrm{r}} \text { observed }=\mathrm{I} .2=\mathrm{IO}^{-3}
\end{aligned}
$$

A large number of transmission experiments from antennas to coils have been made by the radio laboratory of this Bureau in the early part of 1919. In a typical case, where $h_{\mathrm{s}}=2 \mathrm{I}$., $h_{\mathrm{r}}=l_{\mathrm{r}}=1.44$, $N_{\mathrm{r}}=8 ., I_{\mathrm{s}}=3 ., R=7.7, \lambda=700 ., d=4800 ., \delta^{\prime}=0.1, L=541$., microhenries. From (4I) and (20),

$$
\begin{aligned}
& I_{\mathrm{r}} \text { calculated }=24 . \times 10^{-6} \\
& I_{\mathrm{r}} \text { observed }=28 . \times 10^{-6}
\end{aligned}
$$

The fact that the observed current is larger than the calculated, in these and other cases, is probably due to the antenna effect, discussed in the next section. The coil structure has capacity, which makes it receive the wave by antenna action in addition to the coil action, thus increasing the current actually received.

Coil to Antenna.-In an experiment made by Dr. Austin, with a large coil at the Bureau of Standards transmitting to the Arlington antenna, $h_{\mathrm{s}}=2 \mathrm{I} .6, l_{\mathrm{s}}=24.4, h_{\mathrm{r}}=122 ., N_{\mathrm{s}}=7 ., I_{\mathrm{s}}=\mathrm{I} ., \quad R=50$., $\lambda=2800$., $d=7800, a=42^{\circ}$. From formula (42),

$$
\begin{aligned}
& I_{\mathrm{r}} \text { calculated }=\mathrm{I} .3 \times 10^{-4} \\
& I_{\mathrm{r}} \text { observed }=\mathrm{I} .5 \times 10^{-4}
\end{aligned}
$$

Coil to Coil.-The only data available to the author on the use of the coil aerial for both transmitting and receiving are from experiments made in I9I7 by Messrs. Kolster, Willoughby, and Lowell, and these are only qualitative. For transmission from a coil at the Bureau of Standards to a portable coil $40 . \mathrm{km}$ away, $h_{\mathrm{s}}=l_{\mathrm{s}}=3 ., \quad h_{\mathrm{r}}=l_{\mathrm{r}}=\mathrm{I} ., N_{\mathrm{s}}=4 ., \quad N_{\mathrm{r}}=15 ., I_{\mathrm{s}}=10 ., \quad R=\mathrm{r} ., \lambda=600$, $d=40$ ooo. The received current calculated from formula (43) is $4.6 \times 10^{-6}$. The observed signal was loud with a simple audion. 
For transmission from a coil located at a lighthouse to a coil on a ship $48 . \mathrm{km}$. away, $h_{\mathrm{s}}=l_{\mathrm{B}}=3.05, h_{\mathrm{r}}=l_{\mathrm{r}}=1.22, N_{\mathrm{s}}=3, N_{\mathrm{r}}=10$, $I_{\mathrm{s}}=1 \mathrm{o}, R=2, \lambda=550, d=48$ o00. The calculated $I_{\mathrm{r}}$ is $1.6 \times 1 \mathrm{O}^{-6}$. The observed signal was audible on a simple audion. Comparing with the current sensibility of an audion stated above, it is seen that both of these results furnish a rough check on the formula.

\section{DISCUSSION OF EXPERIMENTS}

The agreement of the experiments with the theory is highly satisfactory in view of the simple conditions assumed in the theory. The complex practical conditions preclude the likelihood of agreement within a few per cent. The various limitations of the formulas arising from actual experimental conditions are discussed above in Section V, 2.

One characteristic of the experiments with coil aerials is that the observed value of received current is in every case greater than the calculated value. This strongly suggests that the action of the coil structure involves something additional to the pure action as a coil. This would be expected also from theoretical considerations. The inevitable capacities between parts of the aerial circuit must introduce an action analogous to that of an antenna. When it is borne in mind that the coil action is really a second-order effect in comparison with the action of a system of antenna nature, it appears extremely likely that the stray capacities of any coil aerial circuit would introduce an antenna effect which would have to be considered in addition to the pure coil effect. Besides the reasons thus given by theory and by the excessive values of current observed in experiments with coil aerials, there are two other lines of evidence that the antenna effect is not negligible in coil aerials.

One of these additional lines of evidence is furnished by measurements of current in different parts of a coil aerial or the circuit thereof. If the capacities between parts are appreciable, some of the current must leave the conductors and flow off into the dielectric; the current observed with an ammeter must, therefore, be different in different parts of the circuit. These differences are actually found. The fourth kind of evidence that the antenna effect is appreciable in coil aerials is furnished by considerations of radiation resistance, which will now be discussed. Following that, the antenna effect will be considered in more detail.

Radiation Resistance.-It is possible to determine whether in a given system the antenna effect or coil effect predominates by 
measurements of radiation resistance. The radiation resistance has different values and follows different laws for antenna and coil.

Radiation resistance in general is defined by

$$
P=R I^{2}
$$

in which $I$ is the current in the aerial used as a transmitting device, $P$ is the power radiated, and $R$ the radiation resistance. The study of radiation resistance is an important means of facilitating work on aerials. This may be seen from the simple fact that the magnitude of the radiation resistance gives at once the power radiated, and hence the effectiveness of a transmitting aerial or the range of communication can be judged without making transmission experiments. Field tests are thus in large part replaced by laboratory measurements. In addition to this, it is possible to discriminate between the antenna and coil effects.

The magnitude of the radiation resistance of a flat-top antenna, at wave lengths considerably greater than the fundamental, is given by the well-known expression

$$
R_{\mathrm{a}}=\left(39 \cdot 7 \frac{h}{\lambda}\right)^{2}
$$

An approximate expression for the radiation resistance of a coil can be derived very simply, as follows: When a radiated field exists in any part of space, the relation of the power $P^{\prime}$ radiated through that portion of space to the magnetic field intensity there existing is

$$
P^{\prime} \propto H^{2}
$$

for any given distance from the source, whatever the source may be. The total power radiated is proportional to the integral of $P^{\prime}$ over any surface entirely surrounding the source. This integral will be of the same form for $H_{\mathrm{c}}$, the field due to a coil, as for $H_{\mathrm{a}}$, the field due to an antenna, except for the effect of the variation of $H_{\mathrm{o}}$ in a plane around the radiating coil, which varies from zero to the value given in (Io) for any given distance from the source. As a first approximation, this variation may be considered to make the integrated value of $H_{\mathrm{c}}$ one-half as great as it would be if $H_{\mathrm{c}}$ had the value given in (Io) in all directions around the radiating coil.

$$
\therefore \frac{P_{\mathrm{c}}}{P_{\mathrm{a}}}=\frac{\mathrm{I}}{2} \frac{H_{\mathrm{c}}{ }^{2}}{H_{\mathrm{a}}{ }^{2}}
$$


From (8) and (Io), for a given distance from the source and a coil and antenna of same height with same current,

$$
\frac{H_{\mathrm{o}}}{H_{\mathrm{a}}}=2 \pi \frac{l N}{\lambda}
$$

From (56),

$$
\frac{R_{\mathrm{c}}}{R_{\mathrm{a}}}=\frac{P}{P_{\mathrm{a}}}
$$

Hence from (59) and (60),

$$
\frac{R_{\mathrm{c}}}{R_{\mathrm{a}}}=2 \pi^{2} \frac{l^{2} N^{2}}{\lambda^{2}}
$$

Inserting the value of $R_{\mathrm{a}}$ from (57),

$$
R_{\mathrm{o}}=3 \text { I } \text { I00. } \frac{h^{2} l^{2} N^{2}}{\lambda^{4}}
$$

If the coil is a square one with $h=l=a$,

$$
R_{\mathrm{c}}=\left(\mathrm{I} 3 \cdot 3 \frac{a}{\lambda}\right)^{4} \dot{N^{2}}
$$

This approximate expression for radiation resistance of a coil gives at once the variation with size, number of turns, and wave length. For example, for a set of coils of varying size, in which the length of wire is kept constant, $R_{\mathrm{c}} \propto \frac{\mathrm{I}}{N^{2}}$. It shows that for a given ratio of size to wave length, $R_{\mathrm{c}} \propto N^{2}$. The principal point of interest is that $R_{\mathrm{c}}$ is inversely proportional to the fourth power of wave length.

Since the radiation resistance of an antenna is inversely proportional to the second power of wave length, and that of a coil inversely proportional to the fourth power, the radiation resistance furnishes a means of determining whether a given structure functions as a coil or as an antenna. Rough determinations of radiation resistance which were made upon a particular coil aerial showed a variation of observed radiation resistance inversely as the third power of the wave length, thus verifying the idea that the action is a combination of coil and antenna effect. The observed values, however, were all higher than the sum of the theoretical $R_{\mathrm{a}}$ and $R_{\mathrm{c}}$. The measurement of radiation resistance is an extremely difficult operation, and satisfactory methods can not be said to have been developed as yet. 
Antenna Effect.-Since there are differences of potential between various parts of a coil, acting either as a transmitting or receiving aerial, there must be some dielectric current through the space around the coil and between the coil and ground. It follows that there must be some antenna action, porportional to the amount of this dielectric current and the length of path over which it flows, and this will produce a current additional to that produced by the coil action unless the coil structure happens to

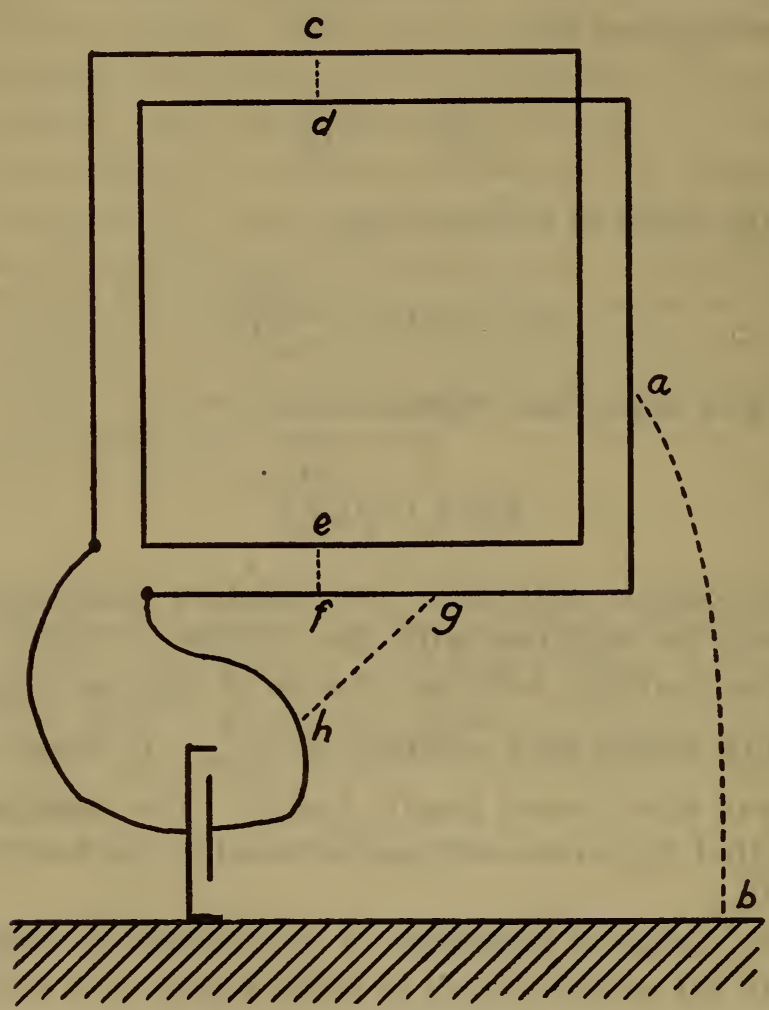

Fig. 16.-Paths of dielectric currents which cause antenna effect in coil aerial

have an exact symmetry which causes the antenna effect in each part of the coil to be balanced by an antenna effect in some other part.

Fig. I6 shows the origin of the antenna effect. As in ordinary practice, the leads cause some part of the apparatus to be practically at ground potential, the shield of the condenser is shown connected to ground. An appreciable dielectric current flows from various parts of the conducting circuit to other parts and to 
ground. Typical paths of this dielectric current are shown by the dotted lines. The line $a b$ suggests the dielectric current from the coil structure to ground, the lines $c d$ and $e f$ the dielectric current between turns of the coil, and the line $g h$ the dielectric current between coil and leads. The flow of dielectric current between turns of the coil is in a horizontal direction when the coil is of prismatic form with the turns separated and all of the same area. This part of the antenna effect arises in a receiving coil of this form only when the wave front is more or less tilted from the vertical.

On account of the flow of current off through the dielectric from various parts of the circuit, ammeters placed at different places in the circuit would show different values of current to be flowing. In radio circuits it can not be assumed that the current is the same at all points around the conducting circuit, as was shown by the author in his investigation of high-frequency current measurement described in Bureau of Standards Scientific Paper No. 206; 1913.

To the extent that these dielectric currents flow, the conductors of the circuit may be considered as an antenna system. Perhaps only the current typified by the line $a b$ might be thought of as giving rise to an "antenna" effect, since the others do not flow to ground; still this part of the dielectric current does not differ from the others in nature or effect, and hence it seems advisable to use the suggestive term "antenna effect" to indicate all of the effect arising from the presence of currents in the dielectric.

It might be supposed that the same sort of an effect would be caused by the flow of dielectric current in the condenser of the coil aerial circuit. This is not true ordinarily, because a condenser of the laboratory type is used in which the condenser plates are interleaved. As shown in Fig. I7, the current in one direction in the dielectric is balanced by a current in the opposite direction in the neighboring part of the condenser. This is a nonradiating condenser and is the analog of a noninductive coil, which is also nonradiating. A condenser consisting of a single pair of plates would radiate, but is not ordinarily used, because it would be much bulkier than the laboratory type of condenser. The condenser consisting of a single pair of plates would be, in fact, the "condenser aerial," which has been recommended by the author in Section IV, 3, as worthy of serious consideration in radio practice. 
The effect of the distributed capacities of the aerial circuit must not be confused in any way with the phase angle between the fields existing at the two vertical sides of the coil aerial. The phase angle referred to is the seat of the action of the coil aerial as such. The dielectric currents flowing in the distributed or stray capacities of the circuit, however, give rise to the direct action as an antenna, not depending in any way on the separation between the two vertical sides of the coil. All of these remarks apply both to transmission and reception.

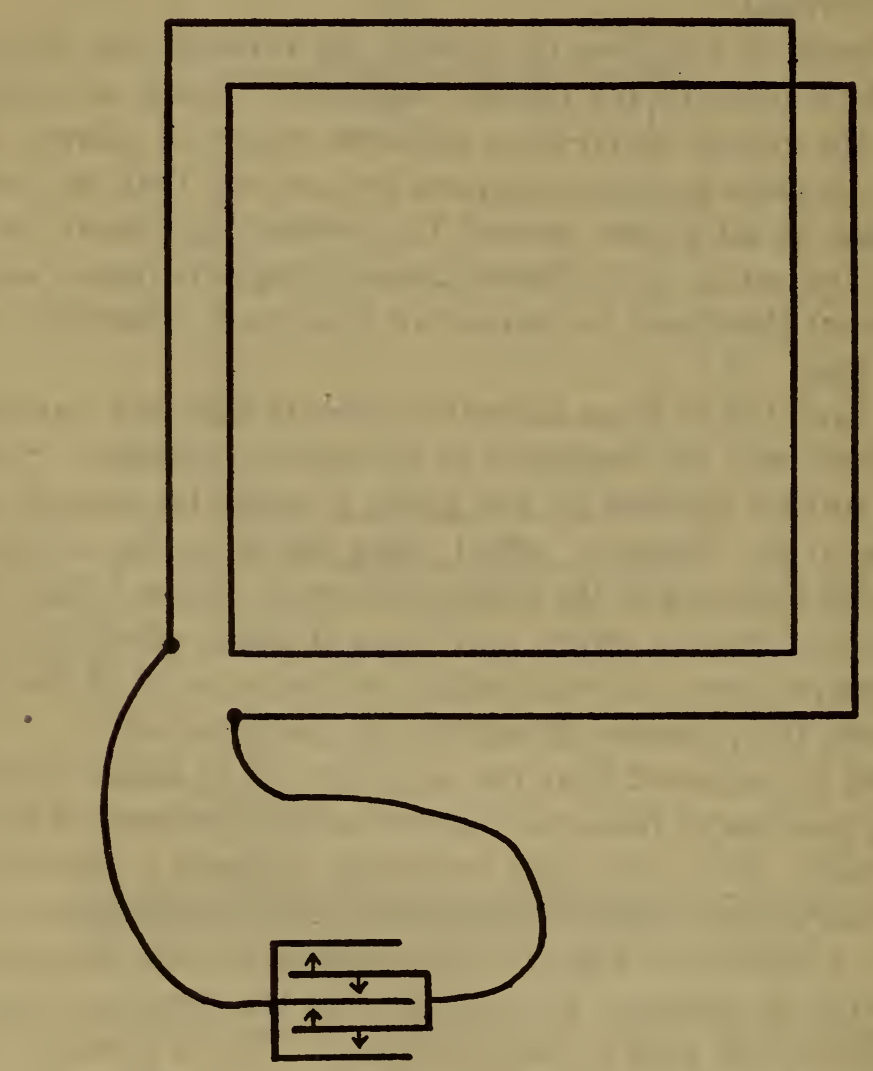

Fig. 17.-Directions of flow of dielectr.c currents in laboratory type of condenser

It is rather difficult to determine what fraction of the effect of a coil aerial is due to antenna action and what part to coil action. In many cases, doubtless, the antenna action predominates. It is possible, however, to separate the effects in any particular case by the several different methods. The antenna effect may be calculated, at least for parts of the circuit, by the aid of careful ammeter readings, which show what amount of the current has flowed off into the dielectric. The antenna effect may be elimi- 
nated, thus leaving only the coil effect, by a carefully-arranged system of shields and grounds; or, by a symmetrical arrangement of the coil structure which causes the antenna effect in each part to be balanced by the antenna effect in some other part. The coil effect may be eliminated, on the other hand, in the case of a receiving coil, by taking advantage of the fact that the coil effect depends on the direction of orientation, while the antenna effect (at least the major part of it) does not; that is, by turning the coil so its plane is parallel to the wave front. A method which eliminates the coil effect and retains a part of the antenna effect is to open one of the coil leads, thus leaving the coil aerial connected to the circuit at one point, placing in series with it an inductance coil of very small dimensions, but of the same inductance, the circuit being completed by the capacity of the coil aerial to ground.

Effects of surroundings.-Currents are induced in metal and other objects near a transmitting aerial, and sometimes are powerful enough to affect the radiation appreciably. The objects near a receiving aerial have currents produced in them by the passing wave. These currents in nearby objects, which may include the ground, induce emf's in the receiving aerial. It is to be noted that this effect of neighboring objects is caused by induction and not radiation from them, which would be comparatively feeble.

The emf thus induced in a coil aerial from the surroundings is of the same or opposite phase as that caused by the wave. It differs in this respect from the emf due to the antenna effect discussed above. The antenna effect ordinarily produces an emf which is $90^{\circ}$ out of phase with the coil effect and does not vary with the orientation of the coil. The antenna effect can thus never balance out the coil effect, and it is impossible to reduce the received current to zero, no matter how the coil is turned. The emf induced by the surroundings, however, depends upon the orientation of the coil. This emf will be reduced to zero by turning the coil at a different orientation from that at which the emf due to the wave is zero unless the line between distorting object and the coil aerial is the direction of propagation of the wave. The result of this is that the total emf is reduced to zero at some orientation other than that obtained when the wave alone acts on the coil aerial. There is thus a distortion in the apparent direction of the wave, caused by objects surrounding the coil aerial. 
Corrections can be made for this effect upon the indications of a direction finder. The errors for various directions of wave are determined, and this gives a calibration by which subsequent observations at the same wave length are corrected.

\section{PRACTICAL CONCLUSIONS}

\section{RELATIVE EFFECTIVENESS OF ANTENNAS AND COIL AERIALS}

(a) Generally speaking a coil aerial is as powerful a transmitting or receiving device as an antenna only when its dimensions approach those of the antenna.

(b) It is easy to make the resistance of a coil aerial circuit much smaller than the resistance of the ordinary antenna circuit and thus make a small coil as effective as a large antenna. A small aerial as effective as a large antenna can, however, also be secured by the use of the antenna-like aerial called the "condenser aerial."

Heeding these principles and using amplifiers in receiving, radio aerials can in the future be much smaller than heretofore.

(c) The relative effectiveness of a coil and antenna, in terms of the wave length, number of turns, etc., is given by formula (33) and the related formulas.

(d) A coil aerial exhibits antenna action as well as coil action, because of capacities between its parts and surroundings. The antenna action sometimes overbalances the coil action.

(e) The advantage of the coil aerial is greatest for short wave lengths. It is consequently likely to be well suited to airplane communication. The increasing advantage of the coil as a transmitting aerial as the wave length is decreased is subject to the proviso that the same current can be gotten into a coil as into an antenna. In fact, the whole practical problem is to get as much current as possible in the aerial.

(f) The use of coil aerials at both receiving and transmitting ends of the communication is particularly suitable for short waves, since the received current in such a system is inversely proportional to the cube of the wave length. 
2. PRINCIPAL FORMULAS

The units used are international electric units, the ordinary electric units based on the ohm, ampere, centimeter, and second, except where otherwise stated. The principal symbols are the following:

\section{Symbols}

$i=$ instantaneous current.

$I_{\mathrm{o}}=$ maximum value of current.

$I=$ effective value of current.

$H_{\mathrm{t}}=$ instantaneous value of magnetic field intensity.

$H_{\mathrm{o}}=$ maximum value of magnetic field intensity.

$H=$ effective value of magnetic field intensity.

$h=$ height of aerial

$d=$ distance along earth's surface from sending aerial.

$\omega=2 \pi$ times frequency of the current.

$t=$ time.

$\lambda=$ wave length.

$c=$ velocity of electric waves $=3 \times 10^{10} \mathrm{~cm}$ per second.

$l=$ horizontal length of coil aerial.

$N=$ number of turns of wire of coil aerial.

$a$ = length of side of square coil.

$\theta=$ phase angle between values of field intensity a distance $l$ apart in the wave.

E $=$ electric field intensity.

$E=$ electromotive force in receiving aerial.

$\delta^{\prime}=$ logarithmic decrement of $H$ or $E$.

$\phi=$ magnetic flux.

$\mathrm{R}=$ resistance of receiving aerial circuit.

$C$ = capacity of receiving aerial circuit.

$L=$ inductance of receiving aerial circuit.

$a=$ angle between direction of propagation of wave and plane of coil.

Subscripts: $\mathrm{s}=$ sending, $\mathrm{r}=$ receiving, $\mathrm{a}=$ antenna, $\mathrm{c}=$ coil.

The following are the principal formulas presented in this paper: Radiated magnetic field intensity from an antenna or condenser aerial:

$$
H=\frac{2 \pi}{\mathrm{IO}} \frac{h_{\mathrm{s}} I_{\mathrm{s}}}{\lambda d}
$$

Radiated magnetic field intensity from a coil:

$$
H=\frac{4 \pi^{2}}{I_{0}} \frac{h_{\mathrm{s}} l_{\mathrm{s}} N_{\mathrm{s}} I_{\mathrm{s}}}{\lambda^{2} d}
$$


Received current in an antenna or condenser aerial:

$$
I_{\mathrm{r}}=300 \cdot \frac{h_{\mathrm{r}} H}{R}
$$

Received current in a coil:

$$
I_{\mathrm{r}}=600 \cdot \pi \frac{h_{\mathrm{r}} l_{\mathrm{r}} N_{\mathrm{r}} H}{R \lambda}
$$

Distance correction factor:

$$
F_{1}=\epsilon-0.000047 \frac{d}{\sqrt{\lambda}}
$$

Decrement correction factor:

$$
F_{2}=\sqrt{\frac{I}{I+\frac{600 \cdot L \delta^{\prime}}{R \lambda}}}
$$

Direction correction factor:

$$
F_{3}=\cos a
$$

Antenna to antenna transmission:

$$
I_{\mathrm{r}}=\frac{\mathrm{I} 88 . h_{\mathrm{s}} h_{\mathrm{r}} I_{\mathrm{s}}}{R \lambda d}
$$

Antenna to coil transmission:

$$
I_{\mathrm{r}}=\frac{\mathrm{II} 84 \cdot h_{\mathrm{s}} h_{\mathrm{r}} l_{\mathrm{r}} N_{\mathrm{r}} I_{\mathrm{s}}}{R \lambda^{2} d}
$$

Coil to antenna transmission:

$$
I_{\mathrm{r}}=\frac{\mathrm{II} 84 \cdot h_{\mathrm{s}} l_{\mathrm{s}} h_{\mathrm{r}} N_{\mathrm{s}} I_{\mathrm{s}}}{R \lambda^{2} d}
$$

Coil to coil transmission:

$$
I_{\mathrm{r}}=\frac{7450 \cdot h_{\mathrm{s}} l_{\mathrm{s}} h_{\mathrm{r}} l_{\mathrm{r}} N_{\mathrm{s}} N_{\mathrm{r}} I_{\mathrm{s}}}{R \lambda^{3} d}
$$

The lengths in the four preceding formulas may be in any units. Meters are commonly used. Any of these formulas may be expressed in terms of $d$. For example, 
Distance at which a given current is received in a coil for a given transmitting current in an antenna:

$$
d=\frac{\mathrm{II} 84 \cdot h_{\mathrm{s}} h_{\mathrm{r}} l_{\mathrm{r}} N_{\mathrm{r}} I_{\mathrm{s}}}{R \lambda^{2} I_{\mathrm{r}}}
$$

Total magnetic field from an antenna, including radiation and induction:

$$
H=\frac{2 \pi}{10} \frac{h_{\mathrm{s}} I_{\mathrm{s}}}{\lambda d}+\frac{j}{10} \frac{h_{\mathrm{s}} I_{\mathrm{s}}}{d^{2}}
$$

Relative effectiveness of coil and antenna for same height and wave length:

$$
\frac{d_{\mathrm{c}}}{d_{\mathrm{a}}}=N \sqrt{2\left(\mathrm{I}-\cos 2 \pi \frac{l}{\lambda}\right)}
$$

Ditto, $l$ small compared to $\lambda$ :

$$
\frac{d_{\mathrm{c}}}{d_{\mathrm{a}}}=6.28 N \frac{l}{\lambda}
$$

Length of coil aerial equivalent to antenna of the same height.

$$
l=0.16 \frac{\lambda}{N}
$$

Current in aerial circuit:

$$
I_{\mathrm{r}}=\frac{E}{R}
$$

Voltage across condenser in aerial circuit:

$$
V=\frac{E}{R \omega C}
$$

Coil aerial reception factors:

$$
\text { Emf reception factor }=\frac{a^{2} N}{\lambda}
$$

Current reception factor $=\frac{a^{2} N}{R \lambda}$

Voltage reception factor $=\frac{a^{2} N L}{R \lambda^{2}}$ 
Radiation resistance:

$$
\begin{aligned}
& R_{\mathrm{a}}=\left(39.7 \frac{h}{\lambda}\right)^{2} \\
& R_{\mathrm{c}}=\left(13.3 \frac{a}{\lambda}\right)^{4} N^{2}
\end{aligned}
$$

\section{FUTURE RESEARCH NEEDED}

The subject of research on electric waves can be considered as barely begun. The study presented in this article has revealed vast and most interesting problems awaiting solution, which can be solved. The functioning of aerials, both in transmitting and receiving, can now be considered as roughly understood. Recent advances in radio measurements and technique open the way to experiments and progress which will bring about far-reaching control of electric waves. A few of the detailed problems which border on the subject matter of this paper and await solution will now be mentioned.

Theoretical Problems.- (a) Develop a simple and straightforward derivation of the radiated field from a coil, directly and without considering the action of the sides separately as antennas.

(b) Work up an explanation of the mechanism of radiation that brings out clearly the relation of the radiation to the induction field and shows that all of the dielectric current is effective in causing radiation, which shall take the place of the usual explanation in terms of the snapping off of lines of force.

(c) Determine the effects of the phase angle between different parts of the dielectric field in an antenna or condenser aerial, especially the long, low types.

(d) Develop methods of measuring radiation resistance.

(e) Work out laws of variation of voltage reception factor of coil aerials, and laws of variation of both current and voltage reception factors of antenna and condenser aerials. Similarly, develop accurate and useful transmission factors.

Experimental Problems.-(a) Determine the relative effectiveness, over a very wide range of sizes, wave lengths, etc., of the various types of aerials. Do this by (I) direct measurements to verify transmission formulas, (2) measurements of the factors that enter into the reception factors, (3) measurements of radiation resistance.

(b) Make transmission experiments at very great distances over typical kinds of land, to obtain distance absorption factors. 
(c) Try out condenser aerials, comparing performance with transmission formulas. Build such aerials with minimum resistance. Demonstrate the nonradiating nature of the laboratory type of condenser, comparing it with condenser aerials.

(d) Compare trailing wire, condenser aerial, and coil aerial, on airplanes.

(e) Find out how directive as transmitting devices coil and condenser aerials and "earth antennas" are; measure magnitude and direction of field at various distances from the aerial, at numerous wave lengths, etc.

(f) Determine relative magnitudes of induction and radiation close to transmitting aerials. Determine also directions of fields, to secure complete knowledge of phenomena near radiating systems.

(g) Measure currents in ground as well as the fields above the ground, to determine how wave attaches itself to the ground.

(h) Study distributed capacities in coil aerial circuits by measuring current at different points in circuit:

(i) Determine values of antenna effect, and develop means of controlling or eliminating it by shielding systems, etc.

(j) Make quantitative investigation of receiving systems combining antenna and coil aerial. Measure phases of currents. Determine under what circumstances the indication of absolute direction is reversed when the tuning is slightly varied.

(k) Determine effects of surrounding objects on currents in transmitting and receiving aerials. Measure magnitude and phase of currents in typical cases.

( $l$ ) Develop methods of connecting generating apparatus to various types of aerials to get maximum current into the aerial, especially at short wave lenghts.

\section{SUMMARY}

The advantages of the coil aerial as a direction finder, interference preventer, reducer of strays, and submarine aerial make it important to know how effective the coil aerial is, in comparison with the ordinary antenna, as a transmitting and receiving device. This article gives the answer. Simple formulas are worked out from fundamental electromagnetic theory, by which the performance of any aerial can be calculated. Experiments have verified the formulas, and show that they are a valuable aid in the choice and design of an aerial to fit any particular requirement. 
The principal formulas are of three kinds: (I) theoretical formulas, giving the magnetic field intensity at any distance from either kind of aerial and the current produced by a given field intensity in either kind of aerial; (2) comparison formulas, giving the ratio of performance of antenna and coil aerial under various conditions; (3) and transmission formulas, giving the current in any receiving aerial in terms of the current in the distant transmitting aerial.

The theory and nature of radiation are discussed, and applied to the elucidation of some current fallacies. There has been a vast haziness of ideas on these points. The distinction between induction fields and radiation fields is presented. It is shown that the receiving action in any kind of an aerial may be considered as arising either from the electrostatic or the magnetic field present in the wave. Such questions are discussed as the distinction between "open" and "closed" circuits. It is shown that a metallically closed circuit can radiate, and that radiation takes place at all frequencies, the amount of radiation being greater the higher the frequency.

The ratio of the range of communication obtainable with a coil aerial to that with an antenna is proportional to the number of turns and horizontal length of the coil and is inversely proportional to the wave length. The coil aerial is hence particularly suited to communication on short wave lengths. A coil aerial is quantitatively as powerful as an antenna only when its dimensions approach those of the antenna. However, it is easy to make the resistance of a coil aerial circuit much smaller than the resistance of the ordinary antenna circuit and thus make a small coil as effective as a large antenna.

A small aerial as effective as the ordinary antenna may be secured without recourse to the coil principle by using an aerial consisting of a condenser having two large parallel plates, arranged so that the dielectric of the condenser includes no ground. 'The circuit of such an aerial may be made to have a very low resistance. It appears likely that, with the use of either condenser or coil aerials together with sensitive amplifiers, radio aerials will in the future be much smaller than heretofore. These principles apply with particular advantage to airplane aerials.

A coil aerial usually functions by a combination of the pure coil action and antenna action. The latter arises from the stray capacities and capacities to ground which are inevitably present. 
The existence of these capacities may be shown by differences in ammeter readings at different points of the circuit. The antenna effect makes the actual received current in experiments with coil aerials larger than the values calculated from the transmission formulas. The observed values are also affected by currents in neighboring objects.

A formula for the radiation resistance of coil aerials is worked out. Comparison of experiment with this formula supplies additional evidence that the coil aerial operates by a combination of antenna and coil effects.

The fundamental principles of design of aerials are given. The various modes of measuring received current and voltage across the condenser are discussed. The relations of these two quantities to the electromotive force acting in the aerial must be carefully observed in calculations or design. Reception factors are derived, to which the received current or voltage are proportional. Experimental data on the functioning of aerials may be secured either from actual transmission experiments or from measurements of the quantities which enter into the reception factor.

This investigation has opened up a large and most interesting field for further research. Progress in the control and utilization of electric waves depends on the investigation of such theoretical and experimental problems as have been suggested in Section VII, 3 , herein.

WASHINGTON, June I8, I9I9. 